\title{
CircNPM1 strengthens Adriamycin resistance in acute myeloid leukemia by mediating the miR-345-5p/FZD5 pathway
}

\author{
JIE DING ${ }^{1 \#}$, XIAOCHUN ZHANG ${ }^{2 \#}$, JIANAN XUE ${ }^{l}, L E F A N G^{3,4}$, CHUNMEI BAN ${ }^{5}$, BIN SONG $^{6}$, \\ LILI WU \\ 'Department of Clinical Laboratory, Jingjiang Chinese Medicine Hospital, Jingjiang, Jiangsu, China \\ ${ }^{2}$ Department of Pediatrics, General Hospital of Ningxia Medical University, Yinchuan, Ningxia, China \\ ${ }^{3}$ Department of Clinical Laboratory, Institute for Hygiene of Ordnance Industry, Xi' an, Shaanxi, China \\ ${ }^{4}$ Department of Clinical Laboratory, The 521 Hospital of Ordnance Industry, Xi' an, Shaanxi, China \\ ${ }^{5}$ Department of Oncology, Liuzhou General Hospital, Liuzhou, Guangxi, China \\ ${ }^{6}$ Department of Hematology, Taihe Hospital (Affiliated Taihe Hospital of Hubei University of Medicine), Shiyan, Hubei, China \\ ${ }^{7}$ Department of Hematology, Fourth Hospital of Hebei Medical University, Shijiazhuang, Hebei, China
}

"Jie Ding and Xiaochun Zhang contributed equally to this work.

\begin{abstract}
Acute myeloid leukemia (AML) is an aggressive hematological malignancy with poor long-term outcomes. Numerous studies claim that circular RNAs (circRNAs) are important regulators in AML progression. This study intended to explore the role of circNPM1 in AML development and drug chemoresistance. The expression of circNPM1 and miR-345-5p was detected by quantitative real-time polymerase chain reaction ( $q R T-P C R)$. Cellular activities, including cell growth, apoptosis, cell cycle, migration and invasion, were monitored using colony formation assay, flow cytometry assay and transwell assay, respectively. The relationship between miR-345-5p and circNPM1 or Frizzled-5 (FZD5) was predicted by the bioinformatics tool starBase and validated by dual-luciferase reporter assay or RNA immunoprecipitation (RIP) assay. CircNPM1 was abundantly expressed in serum samples from AML patients and $A M L$ cell lines. CircNPM1 silence or miR-345-5p restoration repressed colony formation, cell migration and invasion, contributed to cell apoptosis and cell cycle arrest, and weakened Adriamycin (ADM) resistance of AML cells. MiR-345-5p was a target of circNPM1 and was downregulated in AML serum and cells. MiR-345-5p deficiency reversed the effects of circNPM1 silence. Further, FZD5 was targeted by miR-345-5p, and circNPM1 regulated FZD5 expression by adsorbing miR-345-5p. FZD5 overexpression could block the function of miR-345-5p restoration. CircNPM1 might be a vital regulator for ADM chemoresistance in AML cells, which partly depended on the role of the miR-345-5p/FZD5 axis. Our study presents the view that circNPM1 degradation may be a key strategy in AML resistance therapy.
\end{abstract}

Key words: $\operatorname{circNPM1,~miR-345-5p,~FZD5,~AML,~ADM,~chemoresistance.~}$

(Cent Eur J Immunol 2021; 46 (2): 162-182)

\section{Introduction}

Acute myeloid leukemia (AML) is an aggressive hematological malignancy characterized by uncontrolled proliferation of immature myeloid cells [1]. From the genetic background and clinical manifestations, AML is a highly heterogeneous disease [2]. In recent years, with the development of chemotherapy, hematopoietic stem cell transplantation, immunotherapy and molecular targeted therapy, the prognosis of most patients with AML has substantially improved [3]. However, $50 \%$ to $60 \%$ of patients still relapse, without long-term disease-free survival [4]. Studies have shown that drug resistance is the key to treatment failure, which leads to short-term survival of AML [3, 5]. The mechanism of drug resistance in AML is still unclear, which may be the result of a combination of factors [3]. Therefore, it is necessary to explore novel biomarkers for the diagnosis and treatment of drug resistance in AML in order to develop more effective monitoring and treatment programs.

Non-coding RNA (ncRNA) is an emerging tool for diagnosis, prognosis, and treatment of multiple hematological malignancies, including AML [6, 7]. Circular RNA (circRNA) is one class of widely distributed and highly

Correspondence: Lili Wu, Department of Hematology, Fourth Hospital of Hebei Medical University, No. 169, Tianshan Street, Shijiazhuang, 050000, Hebei, China, phone: +86 13472170252, fax: +86 0311-86096328, e-mail: gtsawll@ sina.com Submitted: 21.10.2020; Accepted: 16.02.2021 
stable ncRNA, well known for its circular closure structure [6]. CircRNA is derived from linear mRNA by back-splicing, and its stability is due to the lack of a $3^{\prime}$ and $5^{\prime}$ terminal, so it is more likely to be used as a marker in different human diseases [8]. The involvement of circRNAs is documented in numerous human malignant tumors, including liver cancer, lung cancer and bladder cancer [9]. A growing number of circRNAs are documented to be aberrantly regulated in AML and emerged to be promising biomarkers in AML diagnosis and therapy, such as circANAPC7, circ-VIM and circDLEU2 [10-12]. However, research on circRNAs in AML chemoresistance is still lacking. CircNPM1 (circ_0075001), generated from NPM1, was reported to play a crucial role in AML [13]. We focused on this circRNA and explored its detailed functions in AML cells.

MicroRNA is also a class of ncRNA, approximately 22 nucleotides in length [6]. Widespread dysregulation of miRNAs has been found in AML, and alteration of miRNA expression may trigger AML deterioration or alleviation [6, 14]. Previous reports illustrated that miR-345 was dysregulated in bone marrow from AML patients [15], suggesting that miR-345 might be involved in AML progression. However, related studies that explain the function and mechanism of miR-345 are limited.

Frizzled-5 (FZD5) is a member of the frizzled protein family and serves as a receptor of Wnt proteins [16]. The Wnt signaling pathway is an important axis in tumorigenesis and development, and FZD5 associated with the Wnt pathway participates in the progression of diverse cancers $[17,18]$. Whether FZD5 also plays a key role in AML is worth exploring.

In this study, we investigated the function of circNPM1 in Adriamycin (ADM)-treated AML cells to observe the role of circNPM1 in chemoresistance. Additionally, the related regulatory mechanism of circNPM1 associated with miR345-5p and FZD5 was expounded. Our study aimed to determine the function of circNPM1 in AML and provide new insights into the development of drug resistance in AML.

\section{Material and methods}

\section{Serum samples}

AML patients $(n=20)$ and healthy volunteers $(n=20)$ were recruited from Jingjiang Chinese Medicine Hospital. The peripheral blood samples were collected from each subject, and serums were obtained from peripheral blood by centrifugation. Informed consent was obtained from all subjects before sample collection. Serum samples were preserved at $-80^{\circ} \mathrm{C}$ storage. This study was approved by the Ethical Committee of Jingjiang Chinese Medicine Hospital.

\section{Cell lines and ADM treatment}

Experimental cells, including human AML mononuclear cells (THP-1) and human promyelocytic leukemia cells
(HL-60), and control cells, including marrow stromal cells (HS-5), were all purchased from Bena Culture Collection (BNCC; Beijing, China). THP-1 and HL-60 cells were cultured in 90\% Roswell Park Memorial Institute 1640 (RPMI $1640 ;$ BNCC) $+10 \%$ fetal bovine serum (FBS), and HS-5 cells were cultured in $90 \%$ Dulbecco modified Eagle medium (DMEM; BNCC) + 10\% FBS. All cells were cultured in a $37^{\circ} \mathrm{C}$ incubator mixed with $5 \% \mathrm{CO}_{2}$. Partial cells were exposed to $5 \mu \mathrm{g} / \mathrm{ml}$ ADM for $48 \mathrm{~h}$ before cell transfection.

\section{Quantitative real-time polymerase chain reaction}

Total RNA was extracted from serum samples and cells using TRIzol reagent (Invitrogen, Carlsbad, CA, USA). RNA samples were tested and assembled into cDNAs using the First Strand cDNA Synthesis Kit (Thermo Fisher Scientific, Waltham, MA, USA). Next, qRT-PCR was conducted using SYBR GreenER qPCR SuperMix (Invitrogen) with a PCR system (Bio-Rad, Hercules, CA, USA). Glyceraldehyde 3-phosphate dehydrogenase (GAPDH) and U6 were utilized as internal references. The delta-delta cycle threshold $(\Delta \Delta \mathrm{Ct})$ method was used for quantification. The primers were as follows: circNPM1, F: 5'-GCGCCAGTGAAGAAAGTTGT-3' and R: 5'-AAGGCTGAAATCAAGTAGGGTT-3'; miR-345-5p: 5'-GCTGACTCCTAGTCCA-3' and R: 5'-TGGTGTCGTGGAGTCG-3'; FZD5: 5' - TACCCAGCCTGTCGCTAAAC-3' and R: 5' - AAAACCGTCCAAAGATAAACTGC-3'; U6, F: 5'-CTCGCTTCGGCAGCACA-3' and R: 5'-AACGCTTCACGAATTTGCGT-3'; GAPDH, F: 5' - A GGGCTGCT T T T A ACTGGT-3' and R: 5'-CCCCACTTGATTTTGGAGGGA-3'.

\section{Subcellular distribution}

Cellular fractionation was carried out using the PARIS Kit (Ambion, Austin, TX, USA) to isolate cytoplasmic and nuclear RNA. Cytoplasmic RNA and nuclear RNA were subjected to qRT-PCR to detect the expression of circNPM1 with GAPDH and U6 as the internal references, respectively.

\section{RNase $\mathbf{R}$ treatment}

RNA samples were treated with $3 \mathrm{U} / \mu \mathrm{g}$ RNase R (Epicentre, Madison, WI, USA) for $15 \mathrm{~min}$ at $37^{\circ} \mathrm{C}$ before reverse transcription. After that, RNA was purified using the RNeasy MinElute cleanup Kit (Qiagen, Duesseldorf, Germany) followed by detection using qRT-PCR to determine the expression of circNPM1 and linear mRNA NPM1.

\section{RNA oligonucleotides, plasmids and cell transfection}

Small interference RNA targeting circNPM1 (si-circNPM1) (RiboBio, Guangzhou, China) was used for circNPM1 silence, and si-NC (RiboBio) served as the control. 
MiR-345-5p mimics (miR-345-5p) (RiboBio) or miR-345$5 \mathrm{p}$ inhibitors (anti-miR-345-5p) (RiboBio) were used for miR-345-5p restoration or inhibition, and miR-NC or antimiR-NC served as the respective control. A fusion plasmid of the pcDNA3.1 vector containing the FZD5 fragment (FZD5) (Sangon, Shanghai, China) was used for FZD5 overexpression, and an empty pcDNA vector (vector) (Sangon) served as the control. All of them were introduced into HL-60 and THP-1 cells using Lipofectamine 3000 (Invitrogen).

\section{Colony formation assay}

Transfected THP-1 and HL-60 cells were seeded into 6 -well plates (1000 cells/well) and cultured for approximately 12 days at $37^{\circ} \mathrm{C}$ to allow colony growth. The colonies were fixed with $4 \%$ paraformaldehyde, stained with $0.1 \%$ crystal violet and photographed. The number of colonies was counted under a microscope (Olympus, Tokyo, Japan).

\section{Flow cytometry assay}

Cells were harvested by centrifuging and washed twice with phosphate-buffered saline (PBS). For apoptosis analysis, $1 \times 10^{6}$ cells were treated with $5 \mu$ of Annexin V-FITC and $10 \mu \mathrm{l}$ of propidium iodide from a commercial kit (Beyotime, Shanghai, China), and cultured for $15 \mathrm{~min}$ in the dark. For cell cycle analysis, cells were fixed with $70 \%$ ethanol at $4^{\circ} \mathrm{C}$ for $6 \mathrm{~h}$. Afterwards, cells were exposed to propidium iodide staining buffer containing RNase A $(10 \mathrm{mg} / \mathrm{ml})$ for $30 \mathrm{~min}$ at $37^{\circ} \mathrm{C}$ in the dark. Finally, cell apoptosis or cell cycle distribution at the different stages was analyzed by a flow cytometer (BD Biosciences, San Jose, CA, USA).

\section{Transwell assay}

Cell migration and invasion were monitored using the $8 \mu \mathrm{m}$ transwell chamber (Corning Incorporated, Corning, NY, USA). Partial chambers were treated with Matrigel (Corning Incorporated). $2 \times 10^{4}$ cells resuspended in fresh corresponding culture medium were placed into the upper chambers with or without Matrigel for invasion assay or migration assay, respectively. The lower chamber was supplemented with fresh culture medium to allow cell migration and invasion for $24 \mathrm{~h}$. Eventually, the migrated or invaded cells were fixed using paraformaldehyde and stained with $0.1 \%$ crystal violet followed by photography under a microscope (Olympus).

\section{Western blot}

RIPA lysis buffer (Beyotime) was used for protein extraction. Then protein was treated according to the previous description [12]. The primary antibodies were antimatrix metalloproteinase 2 (MMP2; ab97779; Abcam, Cambridge, MA, USA), anti-matrix metalloproteinase 9
(MMP9; ab76003; Abcam) and anti-FZD5 (ab75234; Abcam). Anti-GAPDH (ab9485; Abcam) was used as a loading control.

\section{Bioinformatics analysis}

The potential target miRNAs of circNPM1 or target mRNAs of miR-345-5p were predicted by the bioinformatics tool starBase (http://starbase.sysu.edu.cn/).

\section{Dual-luciferase reporter assay}

For detecting the interaction between circNPM1 and miR-345-5p, the wild-type sequences of circNPM1 harboring the binding sites with miR-345-5p and its corresponding mutated sequences were inserted into a pmirGLO vector (Promega, Madison, WI, USA), naming them as circNPM1 WT and circNPM1 MUT, respectively. Similarly, the 3' UTR of FZD5 harboring the miR-345-5p binding sites and its corresponding mutated sequence were also cloned into the pmirGLO vector, naming them as FZD5 3' UTR WT and FZD5 3' UTR MUT, respectively. Cells were transfected with these fusion plasmids and miR-345$5 \mathrm{p}$ or miR-NC, respectively. Luciferase activity was determined after 48-h transfection using the Dual-Luciferase Reporter Assay System (Promega).

\section{RNA immunoprecipitation (RIP)}

The Magna RIP Assay Kit (Millipore, Billerica, MA, USA) was used for RIP assay to determine the interaction between circNPM1 and miR-345-5p. According to the protocol, human Argonaute 2 (Ago2) was used for the experimental group, and mouse immunoglobulin $\mathrm{G}(\mathrm{IgG})$ was used as a negative control. After the reaction, the coprecipitated RNAs were extracted and detected by qRT-PCR.

\section{Statistical analysis}

The results were expressed as the mean \pm standard deviation, and the data were calculated from at least three independent experiments. Student's $t$-test was used to evaluate the statistical significance of differences between two groups, and significant differences among $\geq 3$ groups were evaluated using analysis of variance with Tukey's method. All data were processed using GraphPad Prism 5 software (San Diego, CA, USA). A $p$-value less than 0.05 was regarded as statistically significant.

\section{Results}

\section{Expression of circNPM1 was increased in AML}

We collected 20 serum samples from AML patients and healthy volunteers and found that circNPM1 expression was significantly increased in the AML serum compared to the healthy serum (Fig. 1A). Also, the expression of circNPM1 was notably enriched in 
THP-1 and HL-60 cells compared with that in HS-5 cells (Fig. 1B). To further determine the characteristics of circNPM1, we detected its distribution and stability. The data showed that circNPM1 was mainly distributed

A

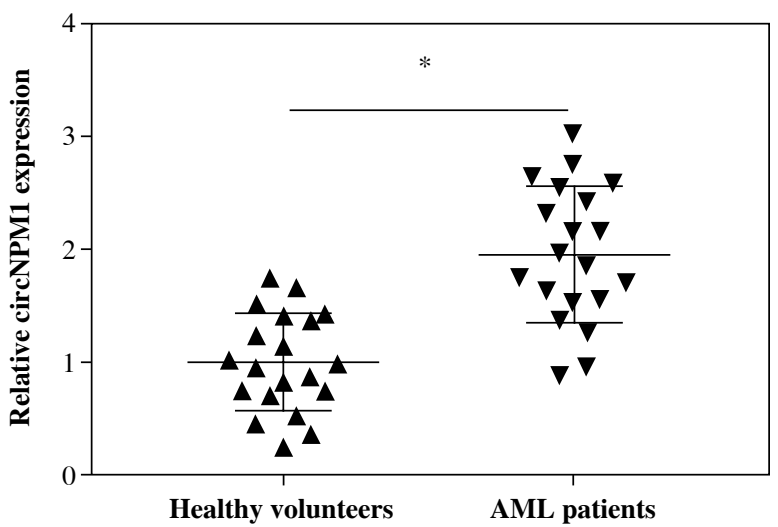

C

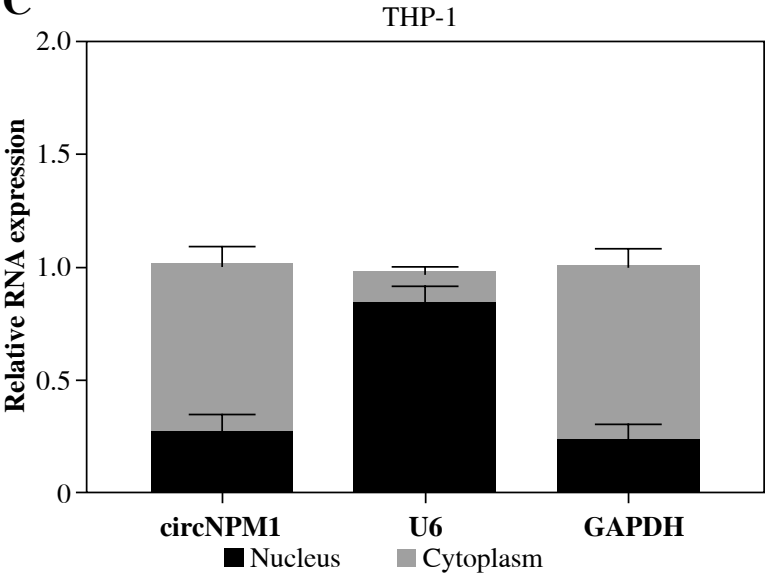

$\mathbf{E}$

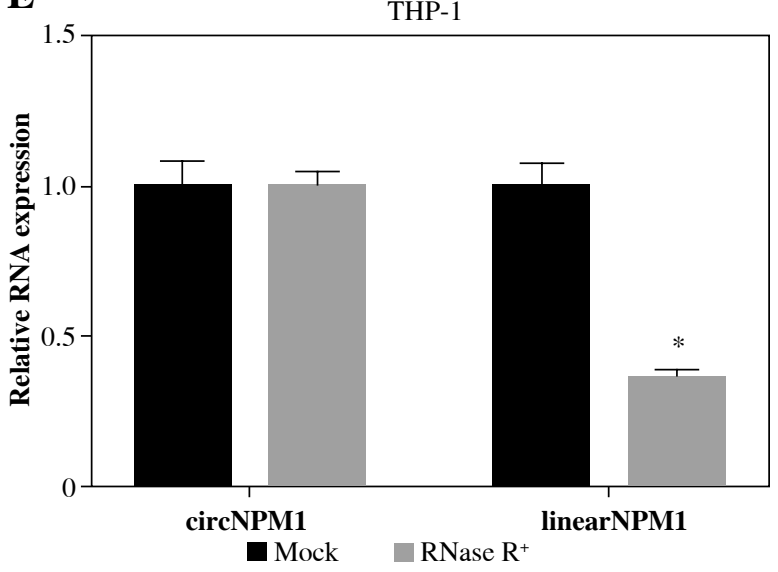

in the cytoplasm rather than in the nucleus (Fig. 1C, D), and circNPM1 was almost unaffected by RNase $\mathrm{R}$ relative to its linear mRNA (NPM1) (Fig. 1E, F). These findings suggested that circNPM1 was highly ex-

B

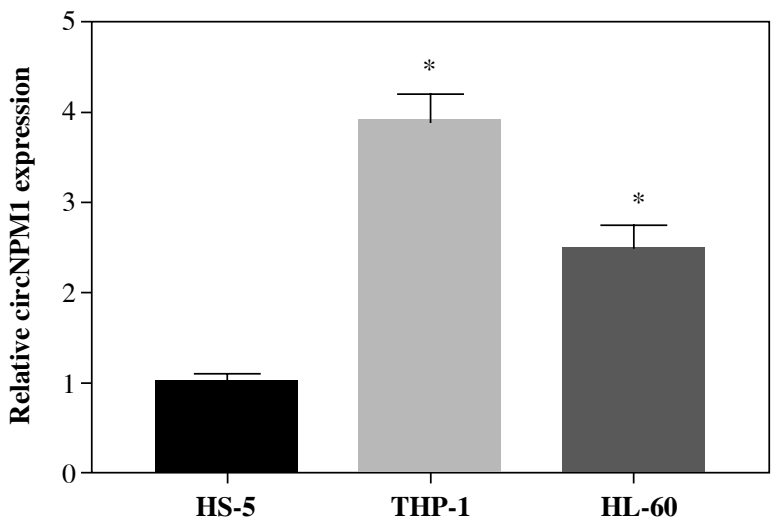

D

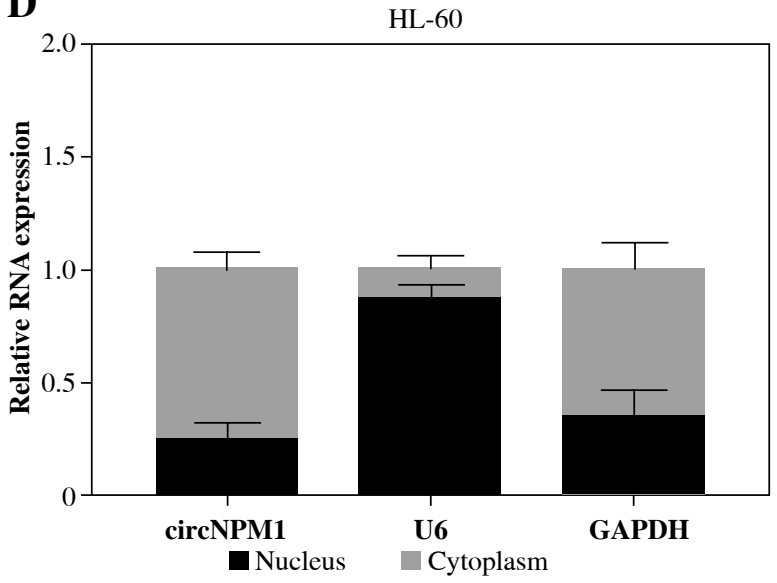

F $\quad$ HL-60

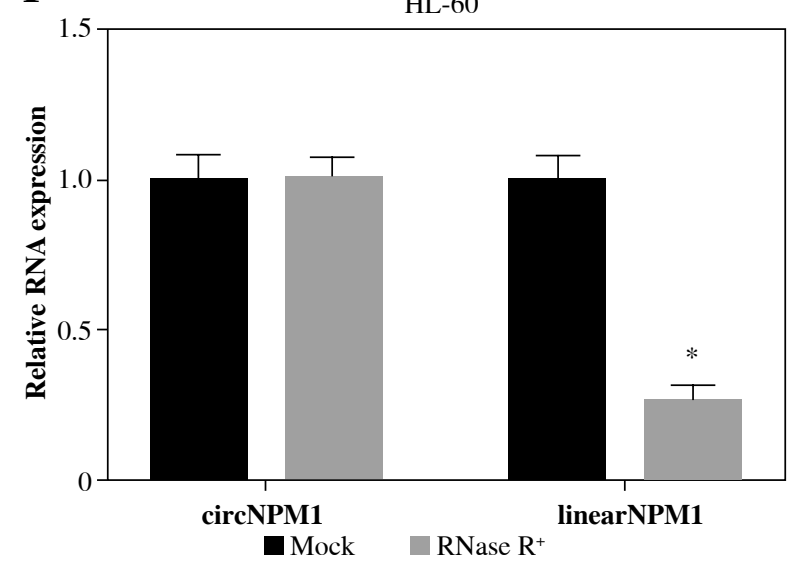

Fig. 1. CircNPM1 was highly expressed in serum from AML patients and AML cells. A) Expression of circNPM1 in serum samples from AML patients and healthy volunteers was detected by qRT-PCR. B) Expression of circNPM1 in HS-5, THP-1 and HL-60 cells was detected by qRT-PCR. C, D) Nuclear and cytoplasmic RNAs were isolated to monitor the distribution of circNPM1 by qRT-PCR. E, F) RNase R was utilized to test the stability of circNPM1 and its linear mRNA. * $p<0.05$ 
pressed in AML and was mainly distributed in the cytoplasm with a degree of stability.

THP-1 and HL-60 cells were subjected to the transfection of si-circNPM1 and si-NC. To ascertain the transfection efficiency, we detected the expression of circNPM1 in transfected cells and found that circNPM1 expression was obviously lower in cells transfected with si-circNPM1 compared to si-NC (Fig. 2A, B), suggesting that the endogenous level of circNPM1 was effectively reduced in cells. For functional analysis, circNPM1 silence signifi-

A

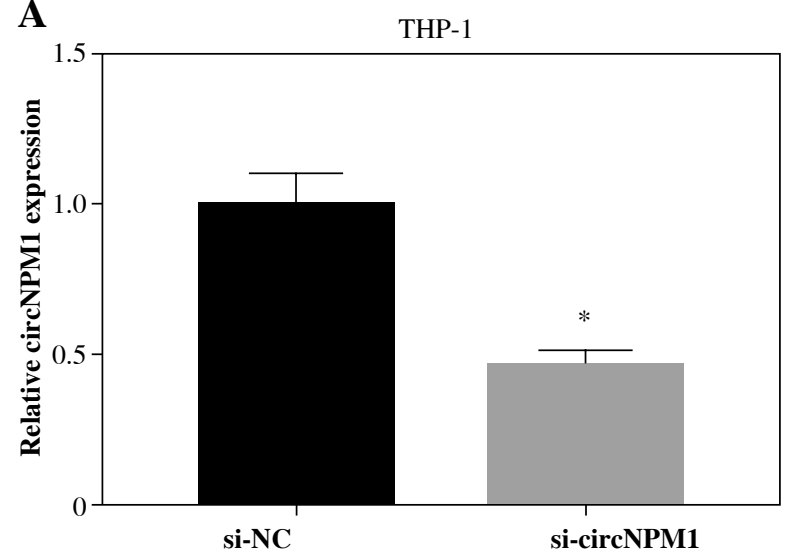

C
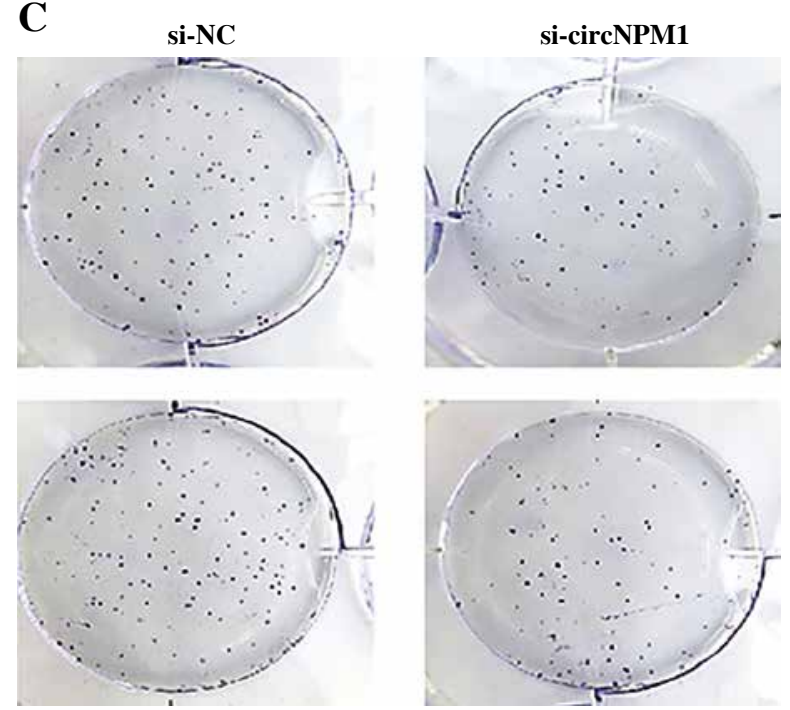

ADM + si-NC

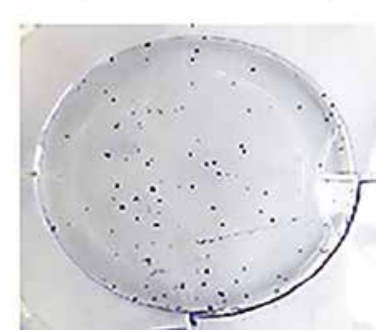

ADM + si-circNPM1

\section{CircNPM1 silence impaired the resistance of AML cells to ADM and inhibited the malignant activities of AML cells}

cantly weakened the ability of colony formation, and the ability of colony formation in cells treated with ADM and transfected with si-circNPM1 was noticeably lower compared with that in cells treated with ADM and transfected with si-NC (Fig. 2C, D). As expected, the apoptosis rate was strikingly higher in cells transfected with si-circNPM1 relative to si-NC, and also enhanced in cells treated with $\mathrm{ADM}$ and transfected with si-circNPM1 relative to si-NC (Fig. 2E, F). Also, circNPM1 silence led to cell cycle arrest at the G1-S transition stage, and si-circNPM1 transfection could enhance the effect of ADM to further block the cell cycle relative to si-NC transfection (Fig. 2G, H). In addition, the capacity of migration and invasion was markedly lower in cells transfected with si-circNPM1 compared with si-NC, and in the si-circNPM1-transfected cells treated with
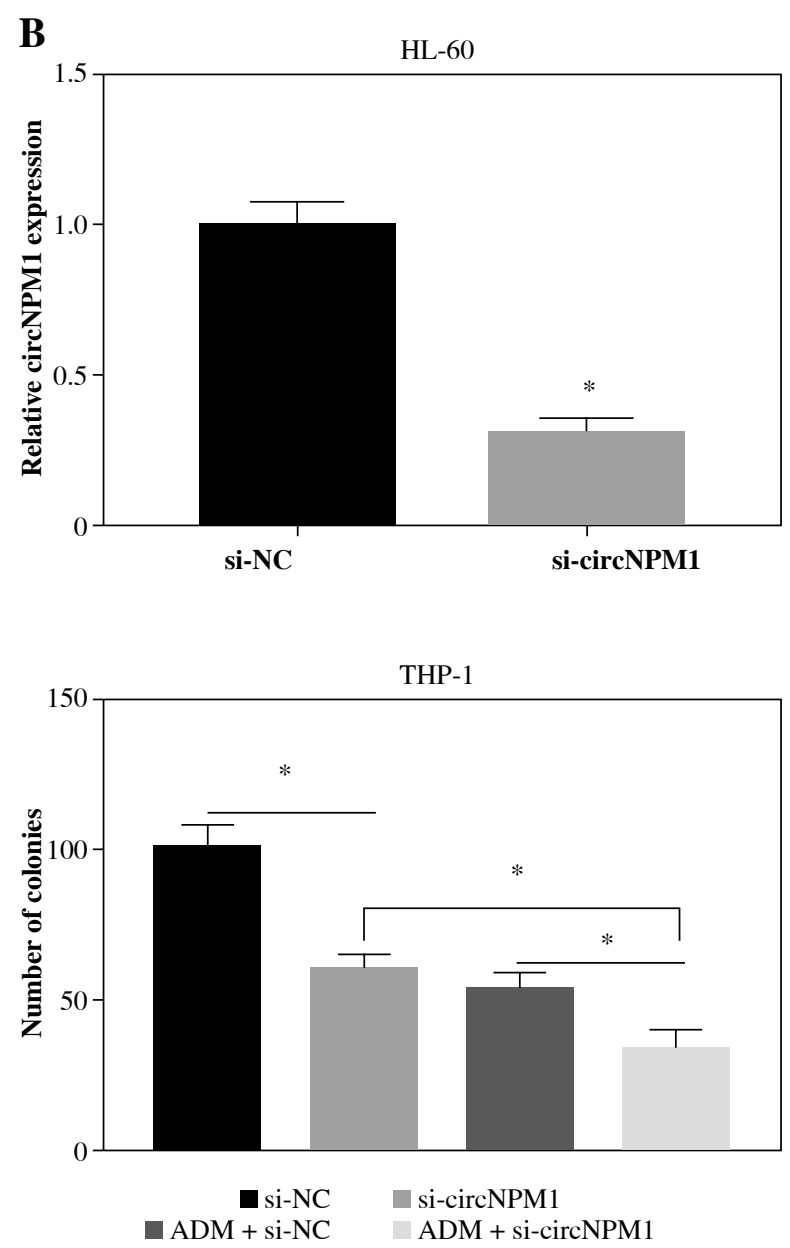

Fig. 2. CircNPM1 silence impaired AML progression and enhanced the sensitivity of AML cells to ADM. A, B) THP-1 and HL-60 cells were transfected with si-circNPM1 or si-NC, and the transfection efficiency was detected according to circNPM1 expression by qRT-PCR. C) Cell proliferation ability was observed by colony formation assay 
D

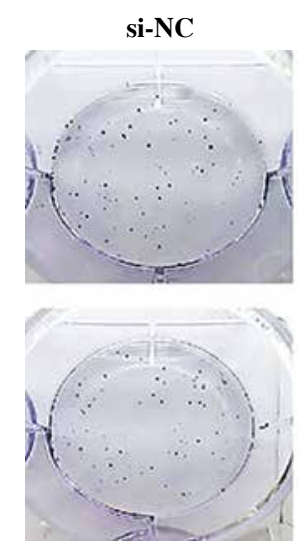

$\mathrm{ADM}+\mathrm{si}-\mathrm{NC}$

$\mathbf{E}$

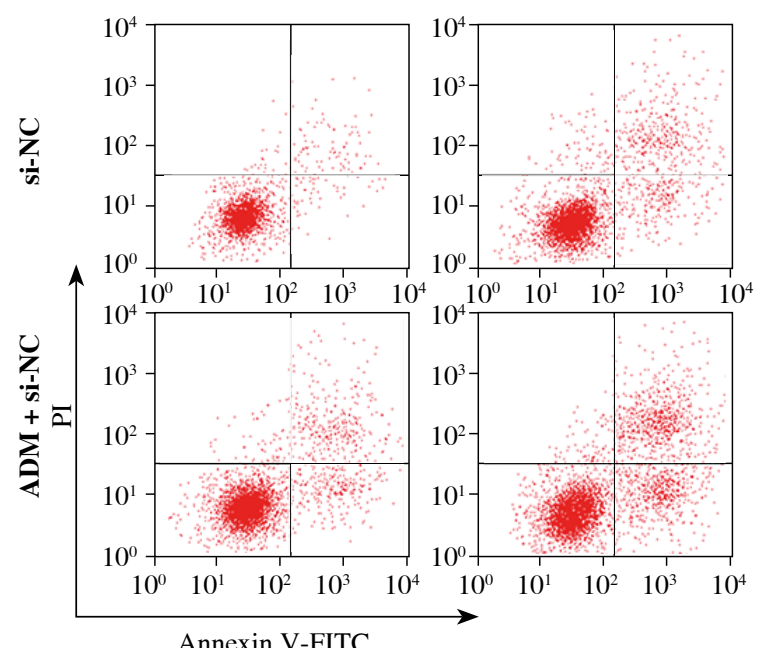

$\mathbf{F}$

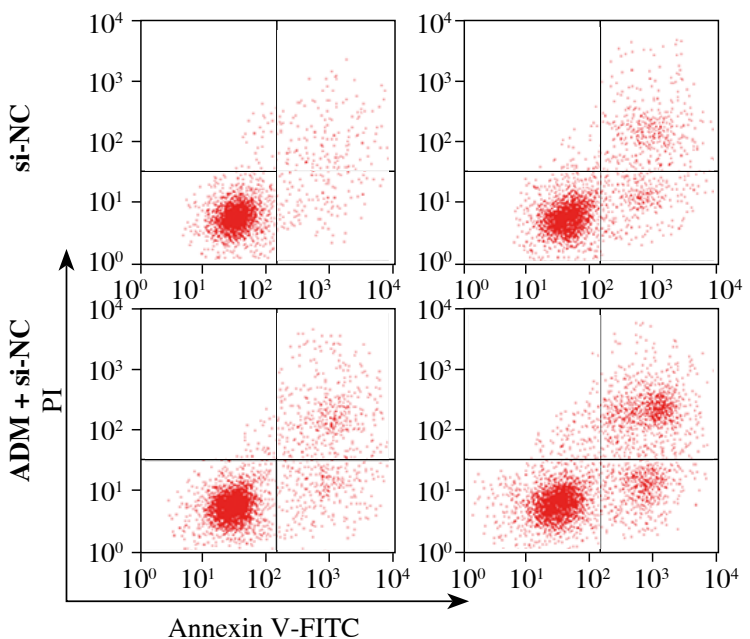

si-circNPM1

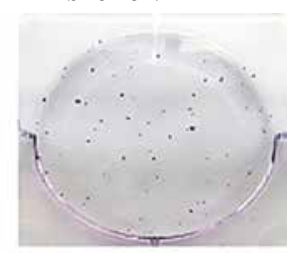

ADM + si-circNPM1
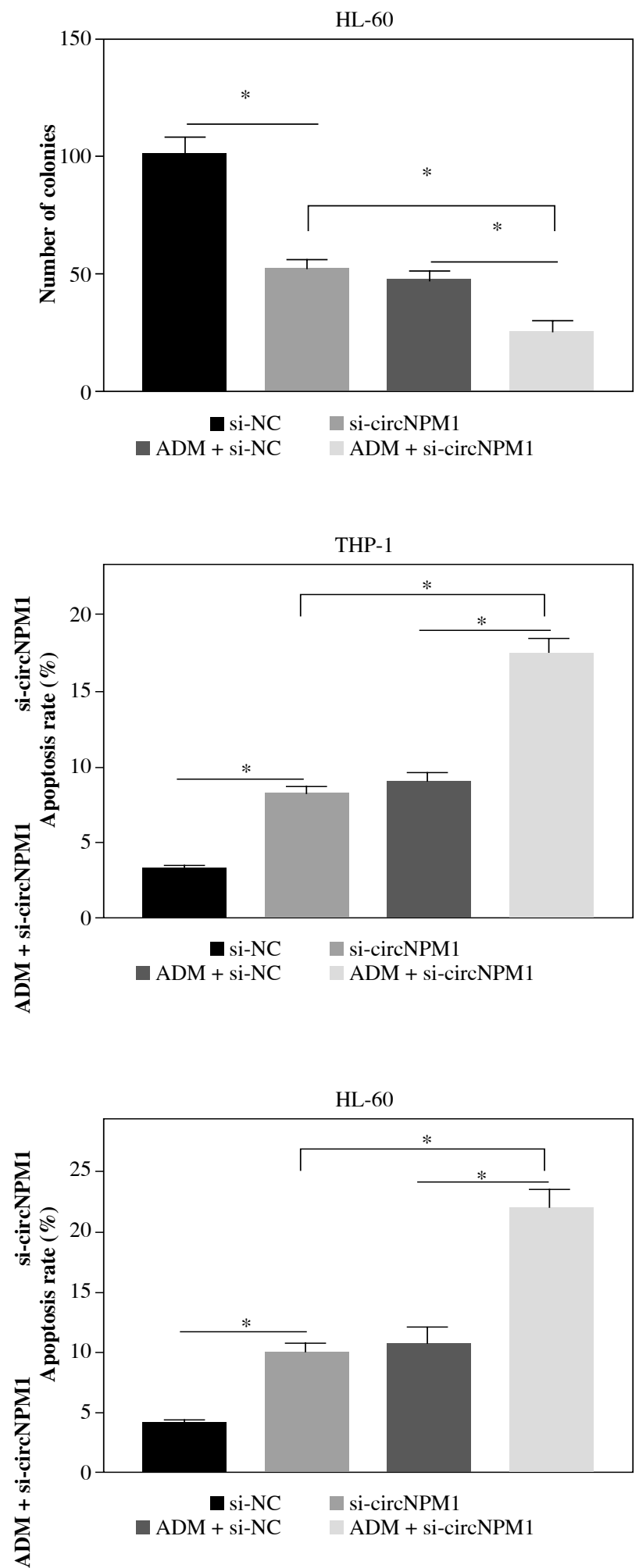

Fig. 2. Cont. D) Cell proliferation ability was observed by colony formation assay. E) Cell apoptosis was monitored by flow cytometry. F) Cell apoptosis was monitored by flow cytometry. 
G
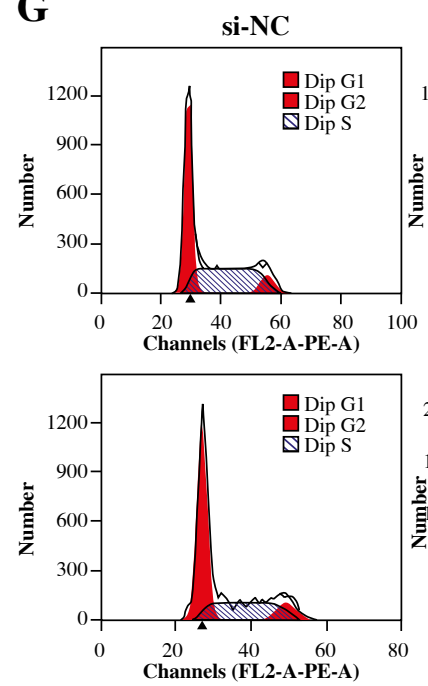

$\mathrm{ADM}+\mathrm{si}-\mathrm{NC}$

H
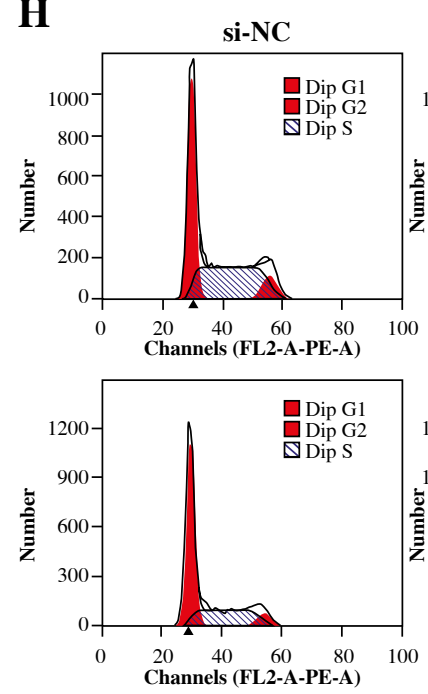

$\mathrm{ADM}+\mathbf{s i - N C}$

I

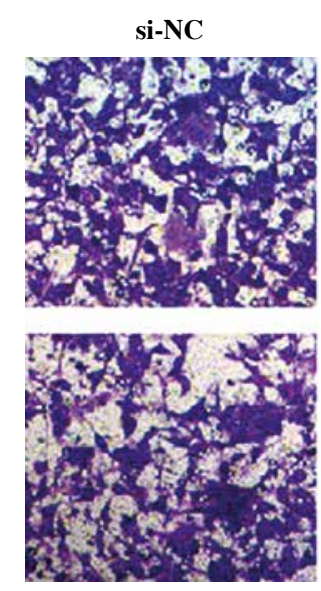

ADM + si-NC
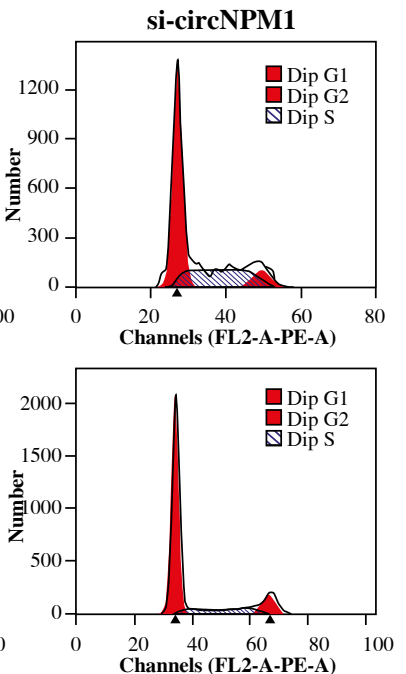

ADM + si-circNPM1
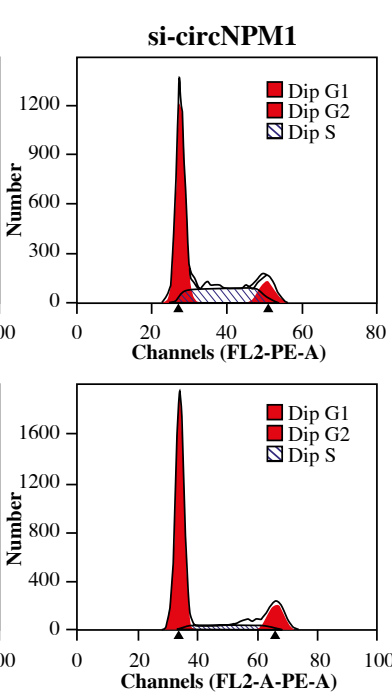

ADM + si-circNPM1
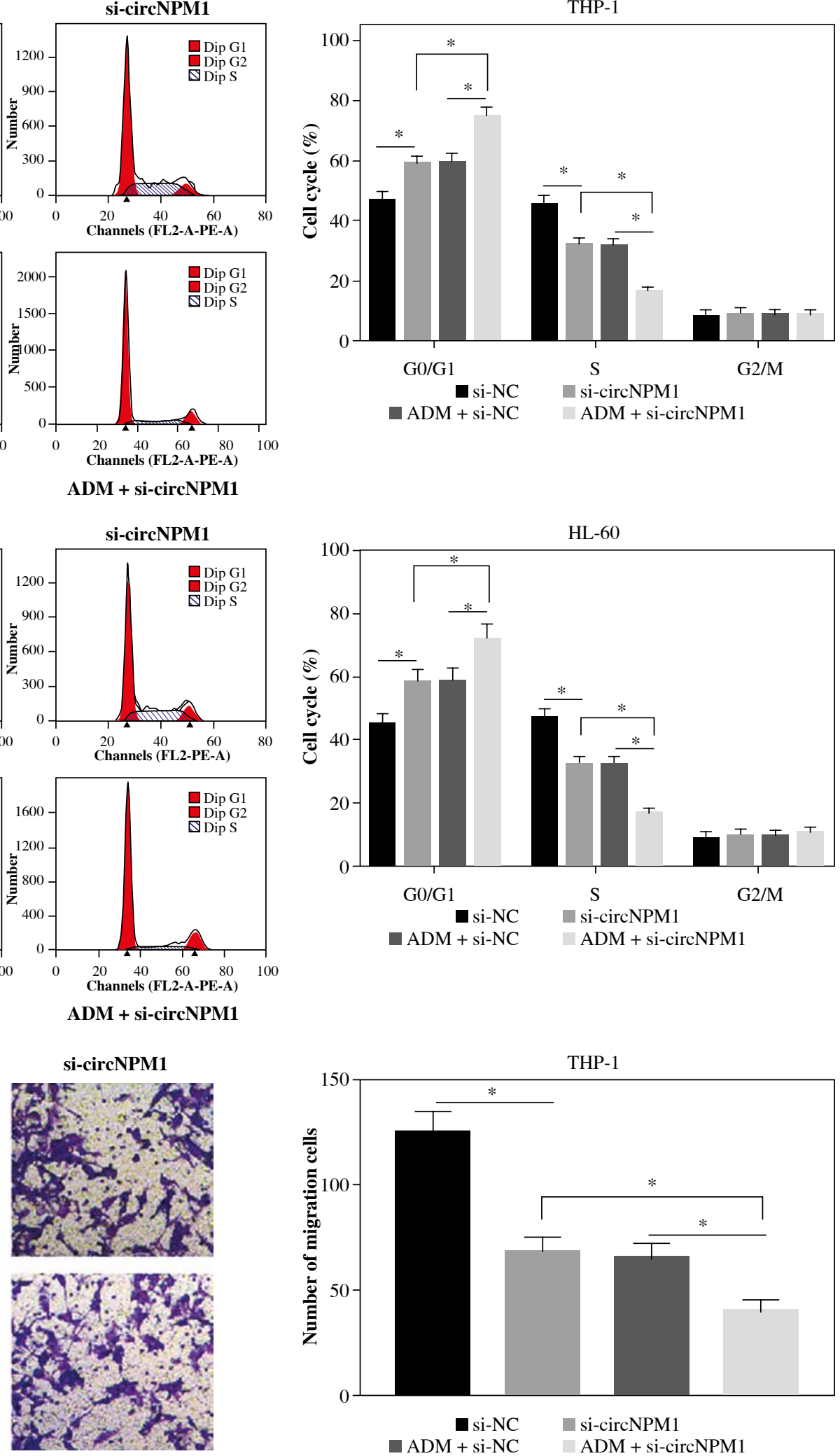

ADM + si-circNPM1

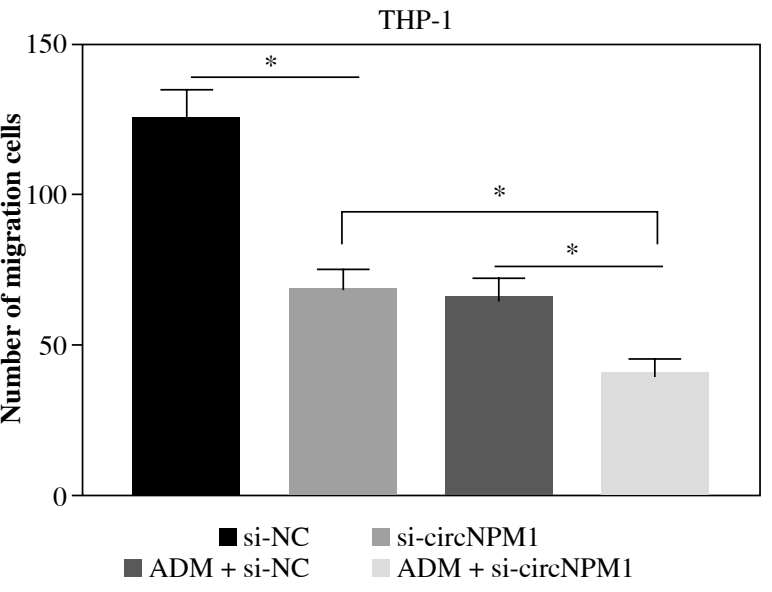

Fig. 2. Cont. G) Cell cycle distribution was assessed by flow cytometry assay. H) Cell cycle distribution was assessed by flow cytometry assay. I) Cell migration and invasion were evaluated by transwell assay 
J
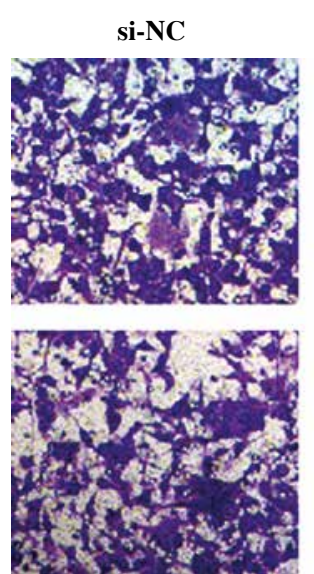

ADM + si-NC

$\mathbf{K}$
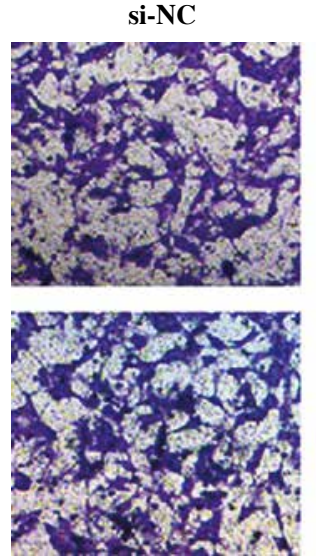

ADM + si-NC

$\mathbf{L}$
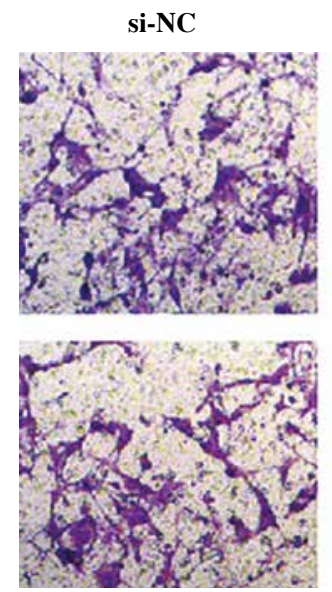

ADM + si-NC
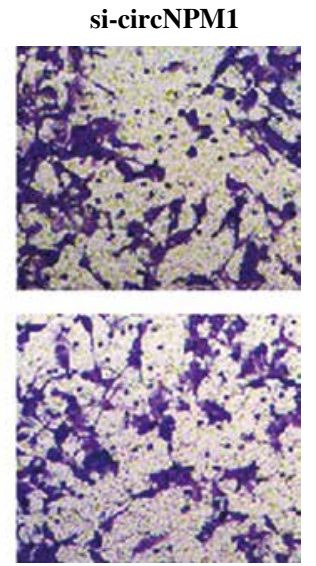

ADM + si-circNPM1

si-circNPM1
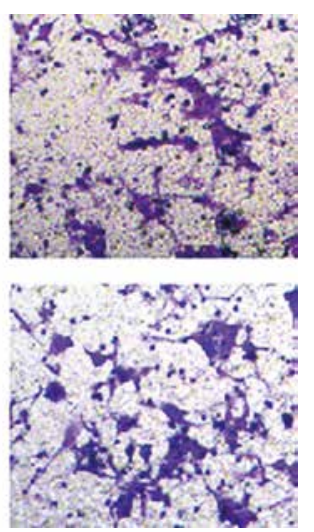

ADM + si-circNPM1

si-circNPM1
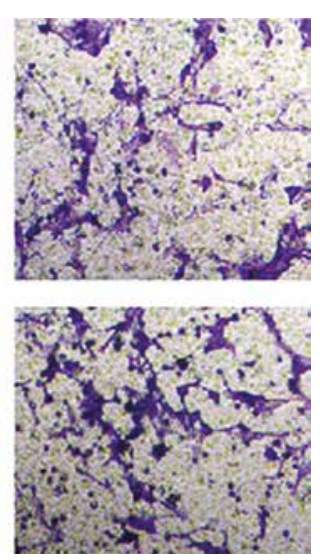

ADM + si-circNPM1
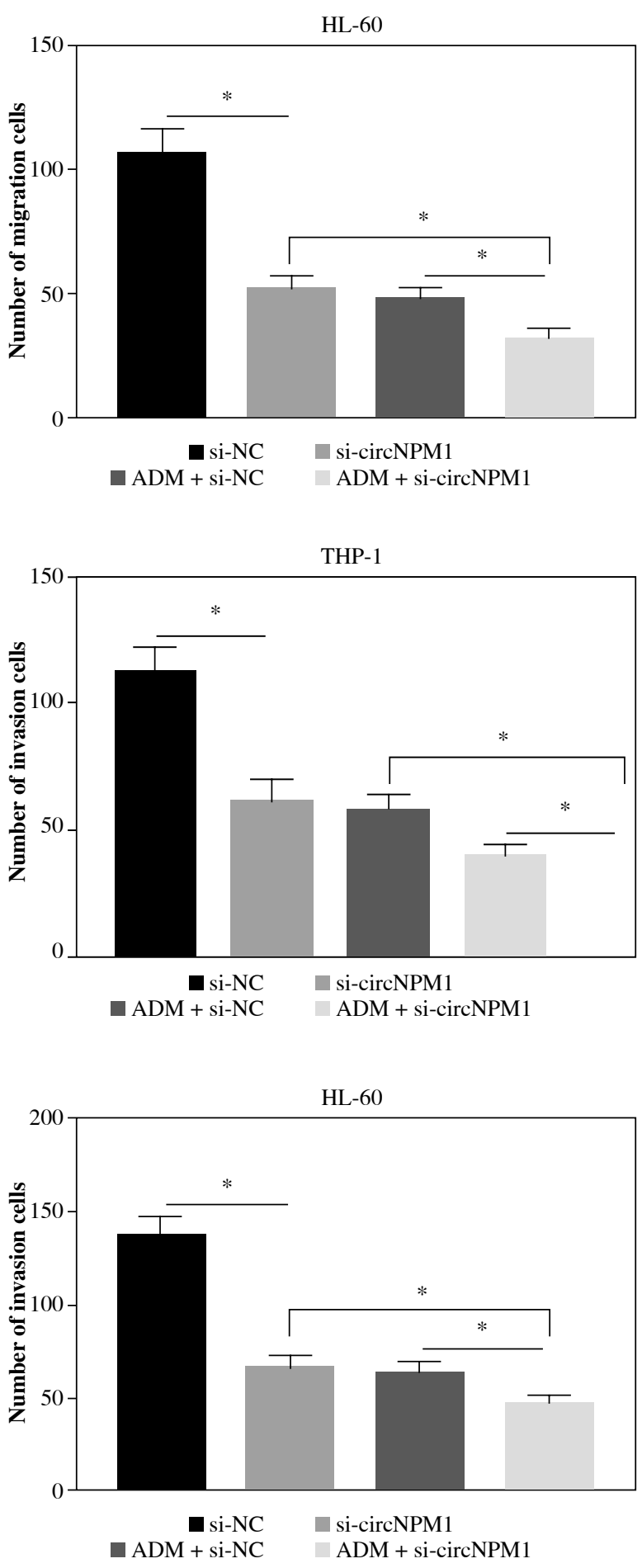

Fig. 2. Cont. J-L) Cell migration and invasion were evaluated by transwell assay 
M

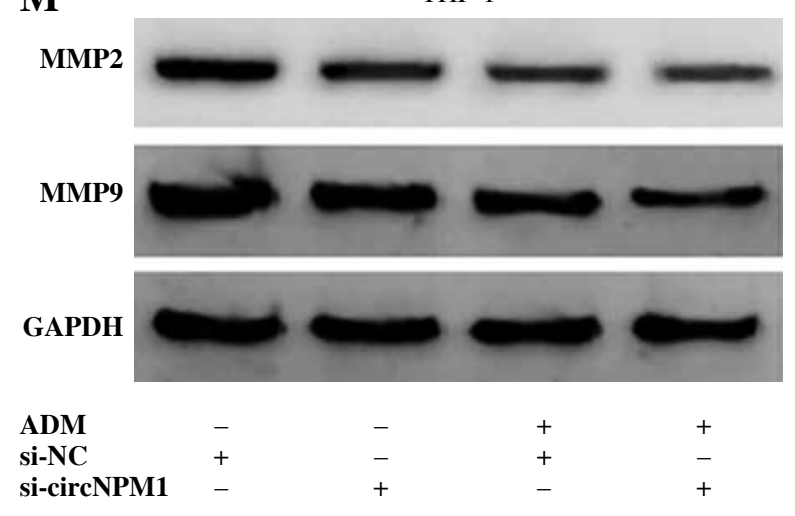

$\mathbf{N}$

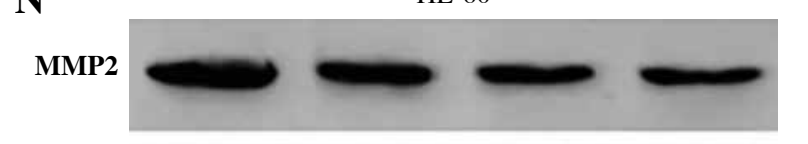

MMP9

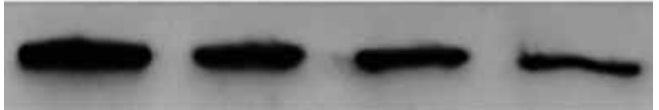

GAPDH

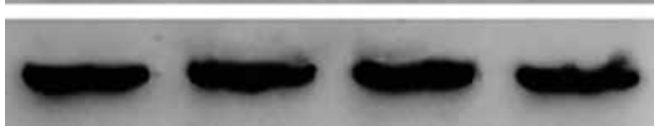

\section{ADM}

si-NC

si-circNPM1

THP-1
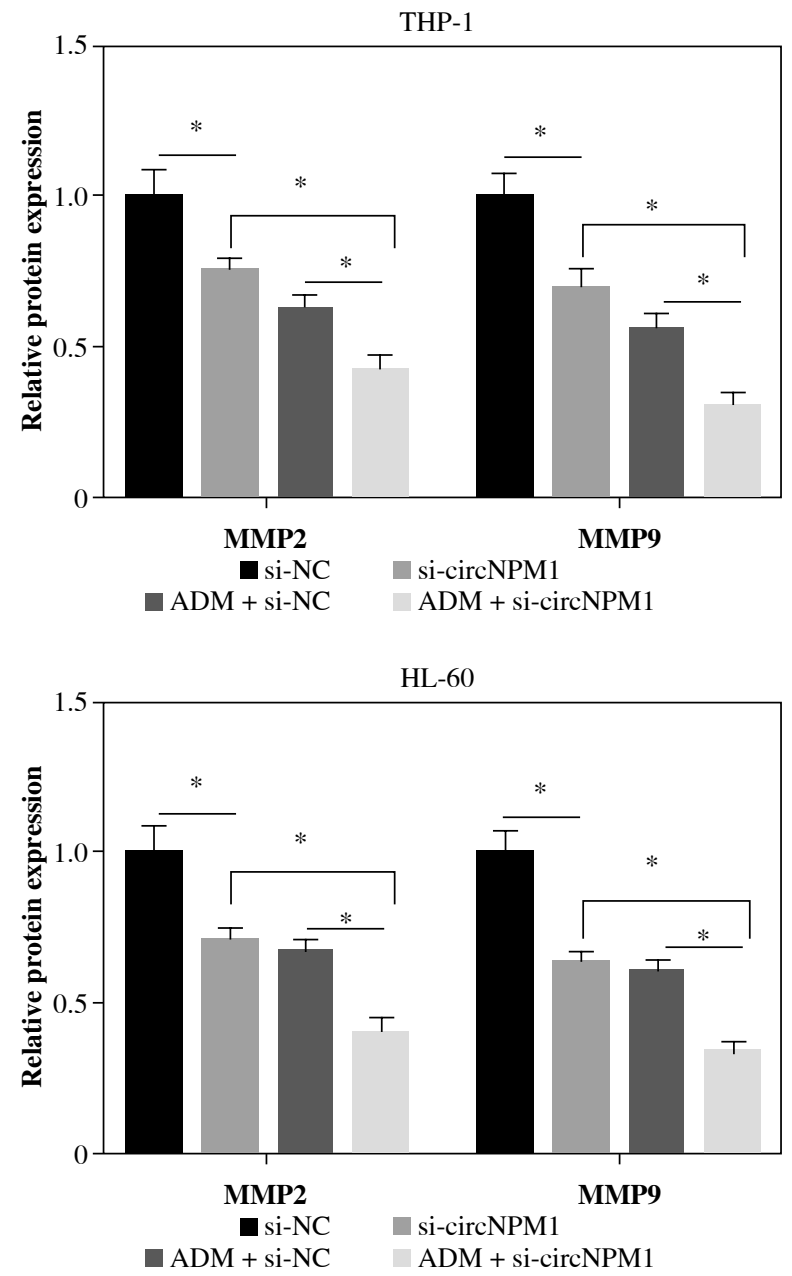

Fig. 2. Cont. M, N) Expression of MMP2 and MMP9 was measured by western blot. * $p<0.05$

ADM, the migration and invasion potential was significantly lower compared to the si-NC-transfected cells treated with ADM (Fig. 2I-L). Additionally, we detected that the expression of MMP2 and MMP9 was weaker in cells transfected with si-circNPM1 relative to si-NC and also weaker in ADM-treated cells transfected with si-circNPM1 relative to si-NC (Fig. 2M, N). These data showed that circNPM1 silence could suppress the malignant activities of AML cells and enhance the sensitivity of AML cells to ADM.

\section{MiR-345-5p was a target of circNPM1 and was downregulated in AML}

Next, we investigated the potential target miRNAs of circNPM1 so as to address its regulatory mechanism in AML. According to the bioinformatics analysis, miR-345-5p was a potential target of circNPM1 with special binding sites between their sequence fragments (Fig. 3A). To verify this prediction, dual-luciferase reporter assay and RIP assay were performed. The data showed that the luciferase activity was strikingly lower in cells cotransfected with miR-345-5p and circNPM1 WT relative to that in cells cotransfected with miR-NC and circNPM1 WT, while the luciferase activity showed no noticeable change in cells cotransfected with circNPM1 MUT and miR-345-5p or miR-NC (Fig. 3B, C). In addition, RIP assay demonstrated that both circNPM1 and miR-345-5p were abundantly detected in the Ago2 RIP group compared with the IgG RIP group (Fig. 3D, E). Moreover, the expression of miR-345-5p was substantially increased in THP-1 and HL-60 cells transfected with si-circNPM1 (Fig. 3F, G), suggesting that circNPM1 suppressed miR-345-5p expression. We also monitored the expression of miR-345-5p in serum samples and AML cells and found that miR-345-5p was significantly downregulated in the serum from AML patients and AML cells (THP-1 and HL-60) relative to healthy samples and HS-5 cells, respectively (Fig. $3 \mathrm{H}, \mathrm{I}$ ). These findings confirmed that miR-345-5p was a target of circNPM1. 


\section{MiR-345-5p deficiency could reverse the effects of circNPM1 silence}

Rescue experiments were performed to functionally explore the relationship between circNPM1 and miR345-5p. Cells were introduced with different transfection, and the data from qRT-PCR showed that miR-345-5p expression was notably promoted in cells transfected with si-circNPM1 relative to si-NC but obviously reduced in cells transfected with si-circNPM1 + anti-miR-345-5p relative to si-circNPM1 + anti-miR-NC (Fig. 4A, B), suggesting that transfection was effective. Next, we transfected si-circNPM1, si-NC, si-circNPM1 + anti-miR-345-5p or si-circNPM1 + anti-miR-NC into ADM-treated THP-1

A

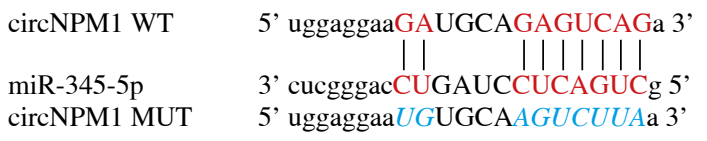

B

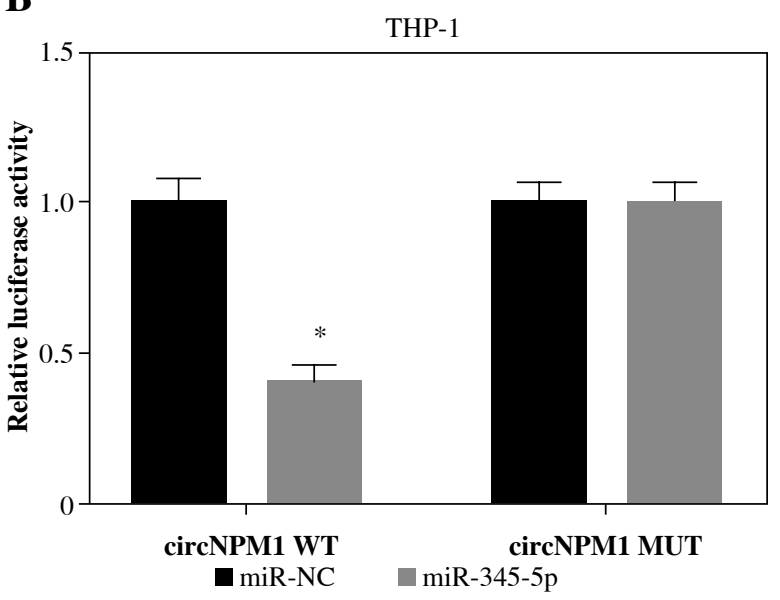

D

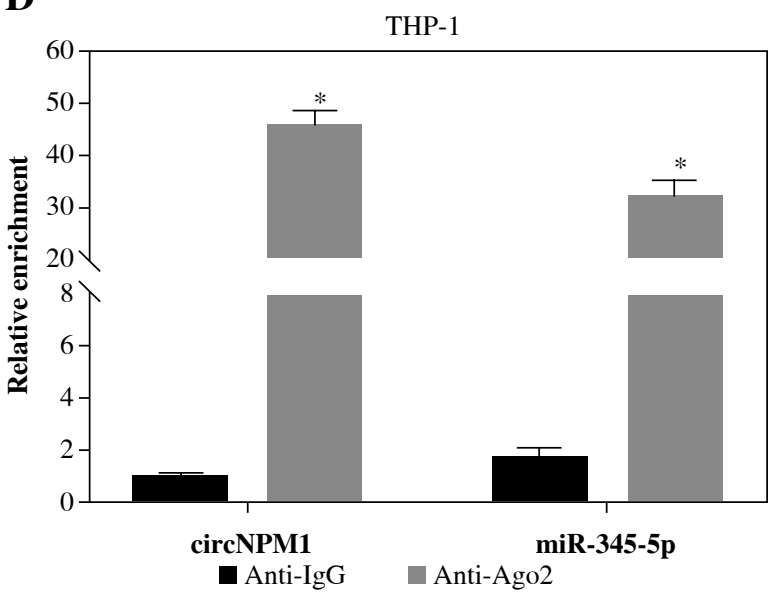

and HL-60 cells, termed the ADM + si-circNPM1 group, $\mathrm{ADM}+$ si-NC group, ADM + si-circNPM1 + anti-miR345-5p group or ADM + si-circNPM1 + anti-miR-NC group. The number of colonies was visibly lower in the $\mathrm{ADM}+$ si-circNPM1 group compared to ADM + si-NC group but recovered in the ADM + si-circNPM1 + antimiR-345-5p group relative to the ADM + si-circNPM1 + anti-miR-NC group (Fig. 4C, D). Also, cell apoptosis stimulated by circNPM1 silence was remarkably reduced by the introduction of anti-miR-345-5p in ADM-treated cells (Fig. 4E, F). Also, cell cycle arrest induced by circNPM1 silence was dramatically relieved by the introduction of anti-miR-345-5p in ADM-treated cells (Fig. 4G, H). Furthermore, cell migration and invasion notably inhibited in the

\section{C}

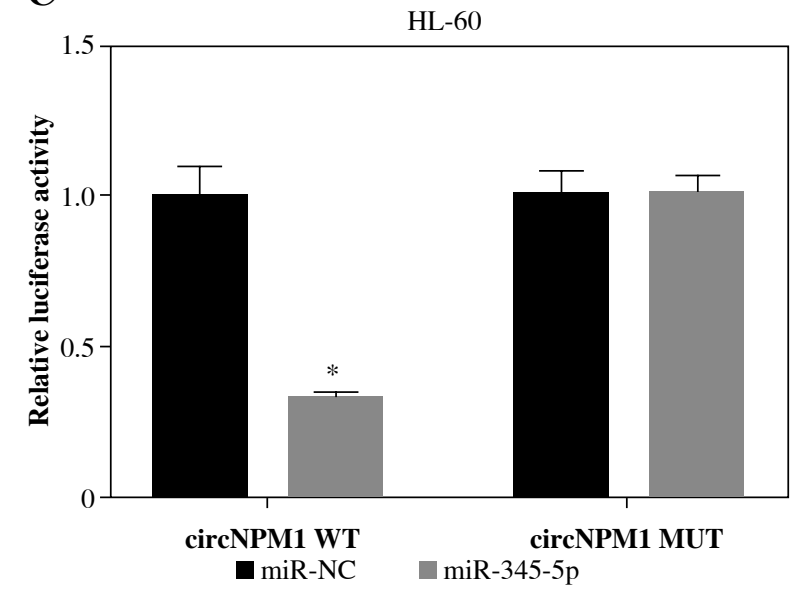

$\mathbf{E}$

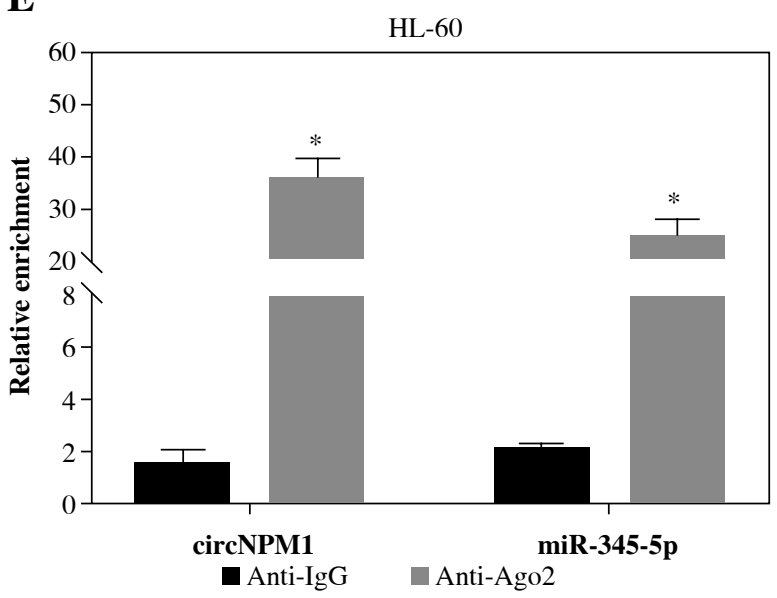

Fig. 3. MiR-345-5p was a target of circNPM1. A) Bioinformatics tool starBase predicted the relationship between circNPM1 and miR-345-5p. B, C) Their relationship was verified by dual-luciferase reporter assay. D, E) Their relationship was also confirmed by RIP assay 
F

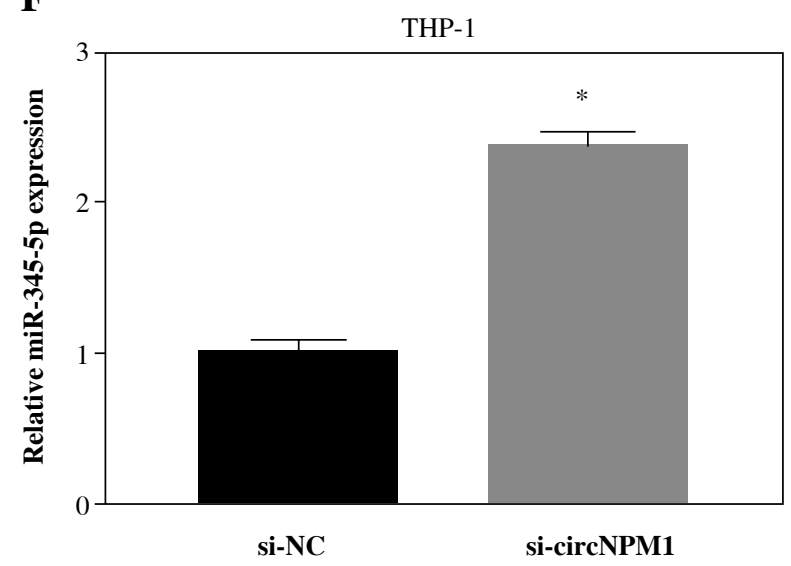

H

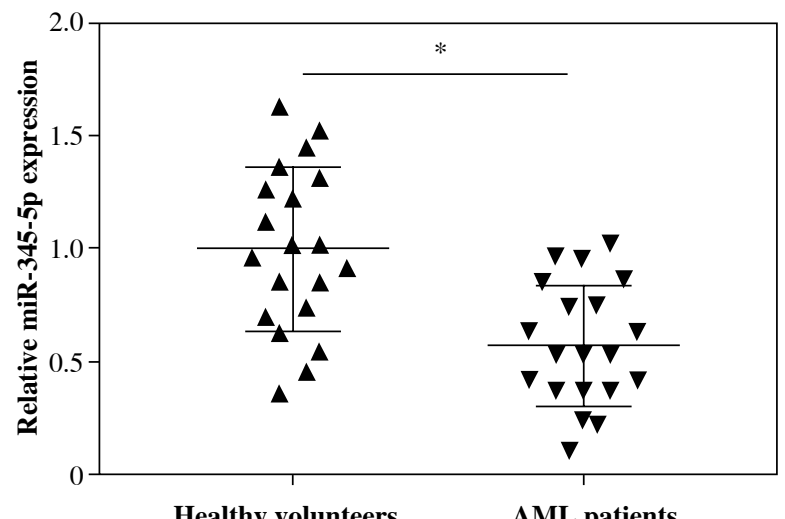

G

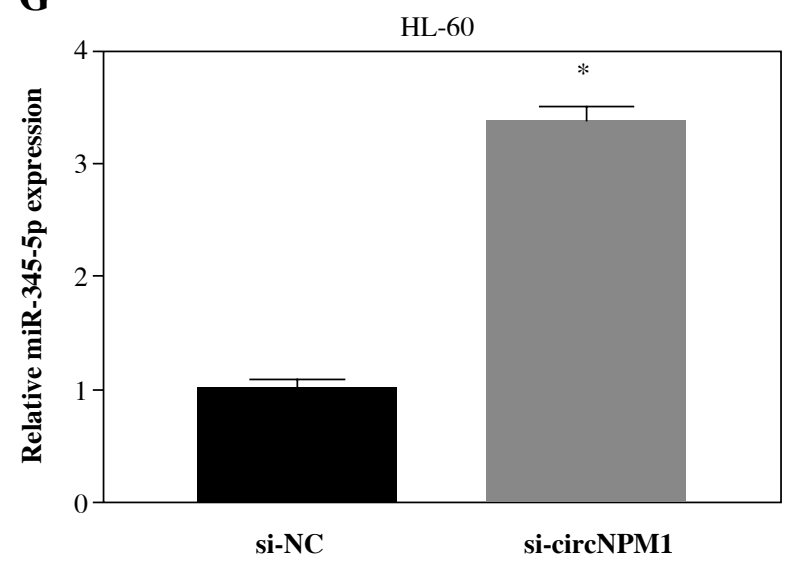

I

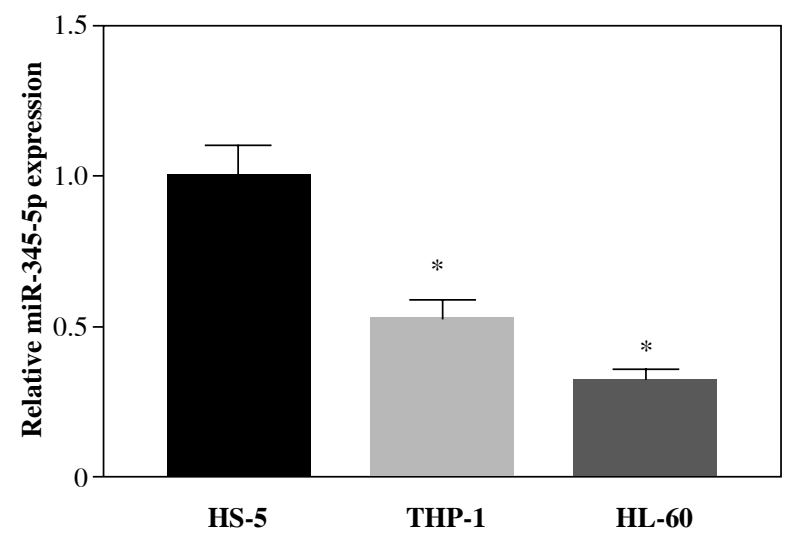

Fig. 3. Cont. F, G) The effect of circNPM1 silence on the expression of miR-345-5p was checked by qRT-PCR. H) Expression of miR-345-5p in serum samples from AML patients and healthy volunteers was detected by qRT-PCR. I) Expression of miR-345-5p in HS-5, THP-1 and HL-60 cells was detected by qRT-PCR. * $p<0.05$

$\mathrm{ADM}+$ si-circNPM1 group were strikingly stimulated in the $\mathrm{ADM}+$ si-circNPM1 + anti-miR-345-5p group (Fig. 4I-L). The expression of MMP2 and MMP9 was prominently lower in the ADM + si-circNPM1 group compared to the ADM + si-NC group but restored in the ADM + si-circNPM1 + anti-miR-345-5p group relative to the ADM + si-circNPM1 + anti-miR-NC group (Fig. 4M, N). These data indicated that miR-345-5p deficiency could partly reverse the functional effects of circNPM1 silence in AML cells.

\section{CircNPM1 targeted miR-345-5p to regulate the expression of FZD5}

Subsequently, the putative target mRNAs of miR-345$5 \mathrm{p}$ were analyzed by the bioinformatics tool. As shown in Figure 5A, there were specific binding sites between FZD5 3'UTR and miR-345-5p sequence fragments (Fig. 5A). Then, the sequence of FZD5 3'UTR was mutated at the binding sites to perform the dual-luciferase reporter assay. The data revealed that miR-345-5p reintroduction notably diminished the luciferase activity in cells transfected with FZD5 3'UTR WT but not FZD5 3'UTR MUT compared to miR-NC (Fig. 5B, C). Also, miR-345-5p enrichment weakened the expression of FZD5 (Fig. 5D, E). Interestingly, we also noted that the expression of FZD5 was significantly lower in THP-1 and HL-60 cells transfected with si-circNPM1 relative to si-NC but higher in cells transfected with si-circNPM1 + anti-miR-345-5p relative to si-circNPM1 + anti-miR-NC (Fig. 5F, G). The expression level of FZD5 in AML serum samples and cells (THP-1 and HL-60) was also aberrantly enhanced compared with that in healthy serum samples and HS-5 cells, respectively (Fig. 5H, I). From the above data, we concluded that circNPM1 regulated the expression of FZD5 by targeting miR-345-5p.

\section{FZD5 could reverse the effects of miR-345-5p}

\section{in AML cells}

To further confirm that FZD5 was a downstream target of miR-345-5p, rescue experiments were performed. 
A

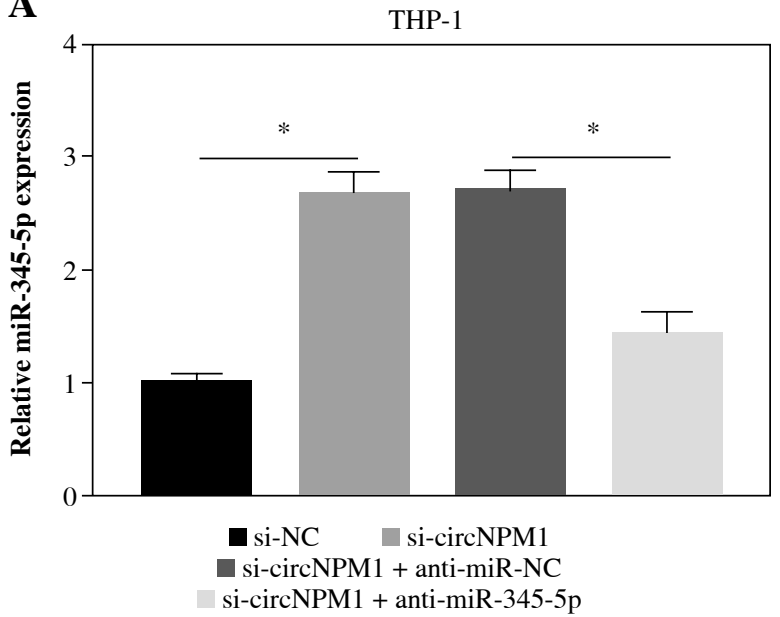

C

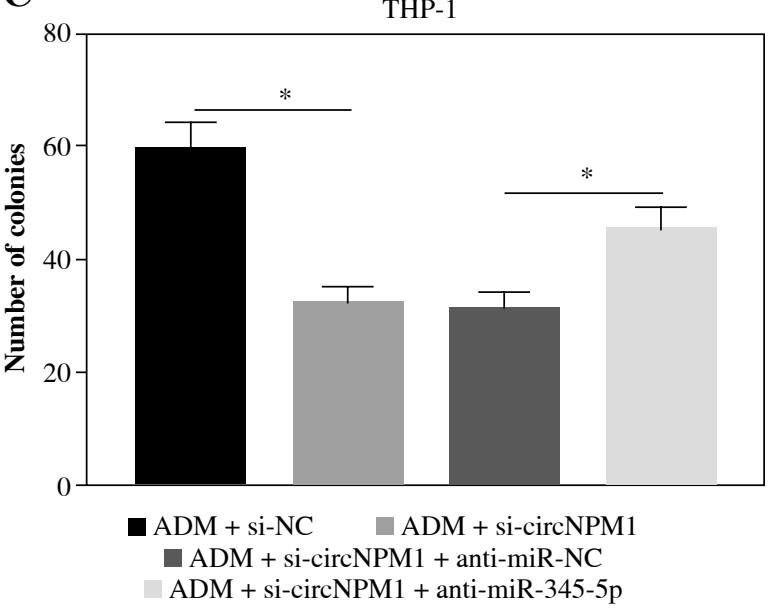

E

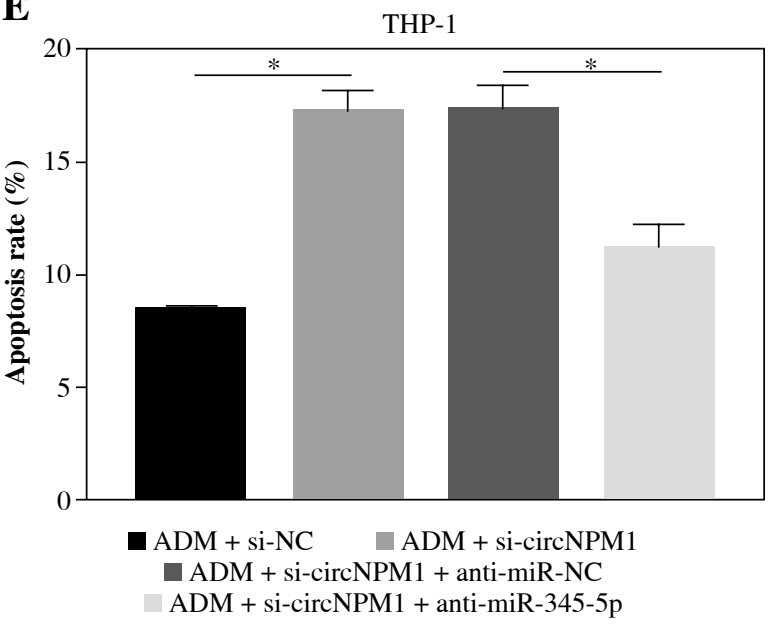

B

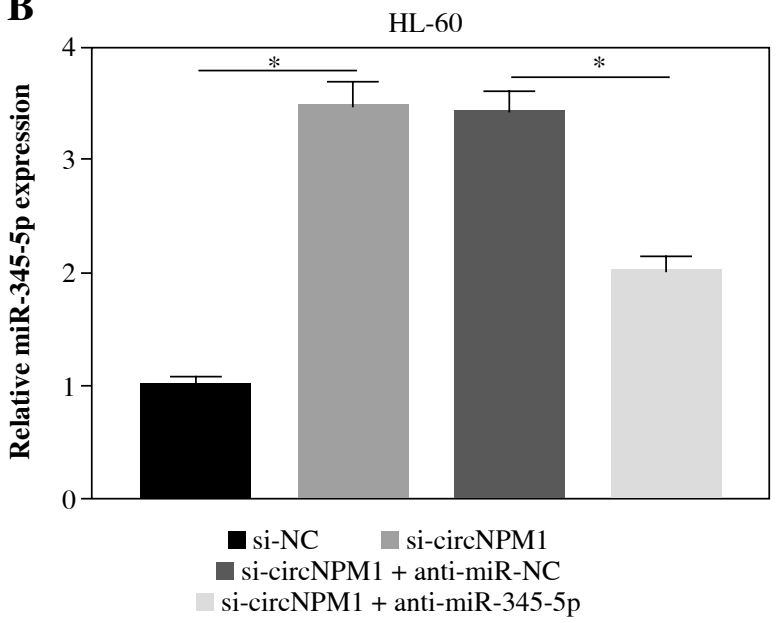

D

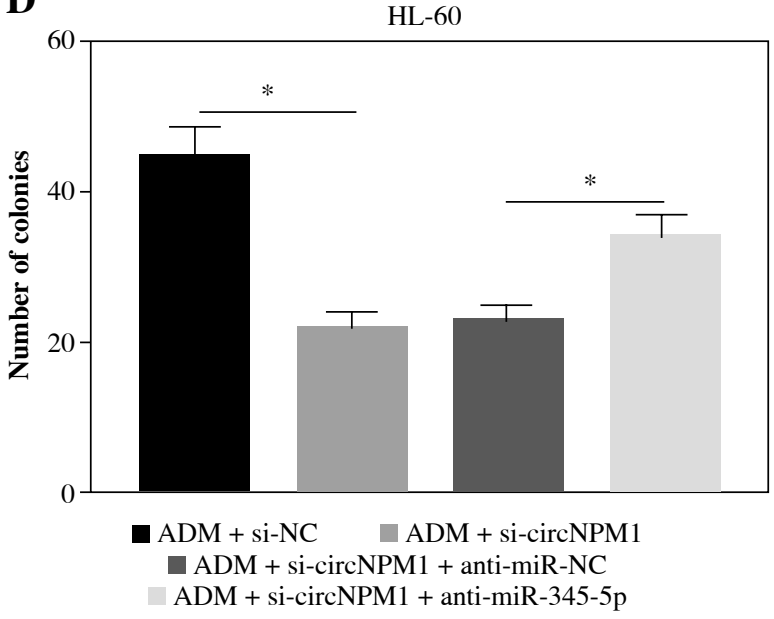

F

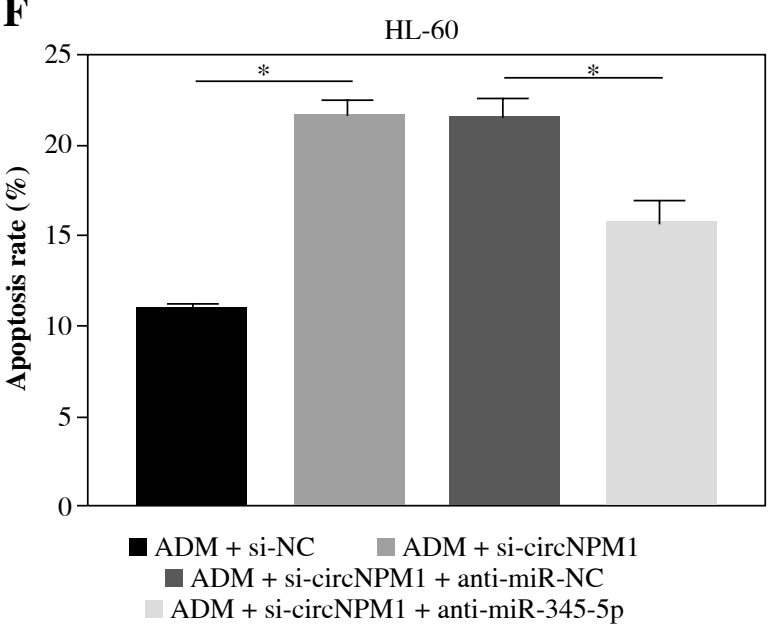

Fig. 4. MiR-345-5p deficiency reversed the effects of circNPM1 silence in ADM-treated cells. THP-1 and HL-60 cells treated with ADM were transfected with si-circNPM1, si-NC, si-circNPM1 + anti-miR-345-5p or si-circNPM1 + antimiR-NC. A, B) The transfection efficiency was checked according to miR-345-5p expression, and cellular activities, including $\mathbf{C}, \mathbf{D})$ colony formation, $\mathbf{E}, \mathbf{F})$ cell apoptosis. $* p<0.05$ 

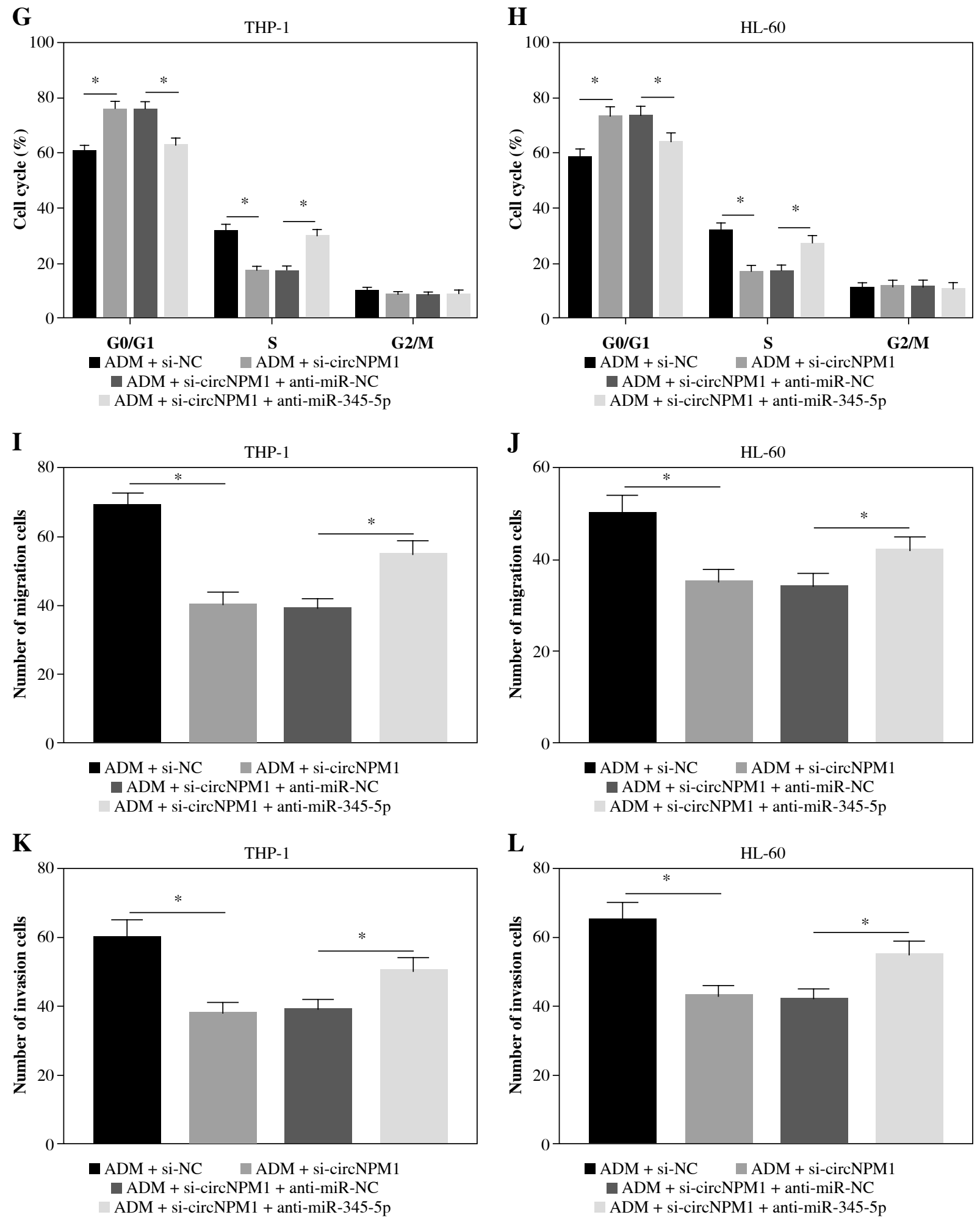

Fig. 4. Cont. G, H) cell cycle, I-L) migration and invasion, were investigated in these transfected cells to observe the function of circNPM1 and miR-345-5p. $* p<0.05$ 

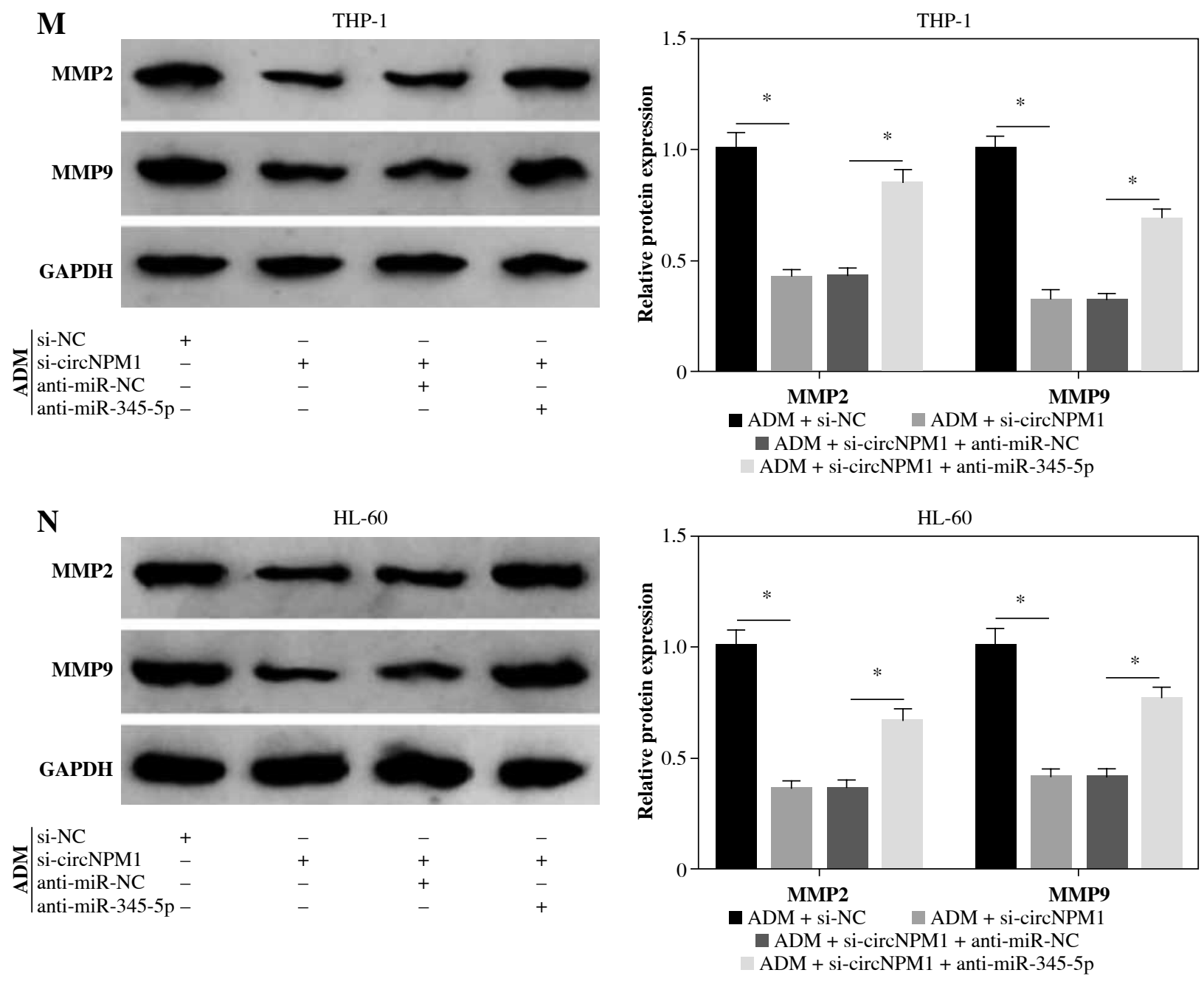

Fig. 4. Cont. M, N) Expression of MMP2 and MMP9 in these transfected cells was detected by western blot. * $p<0.05$

The data from western blot showed that FZD5 expression was blocked in cells transfected with miR-345-5p but visibly recovered in cells cotransfected with miR-345-5p + FZD5 (Fig. 6A, B), explaining that each transfection was available. In ADM-treated THP-1 and HL-60 cells, miR$345-5 p$ transfection alone conspicuously impaired colony formation ability, while combined miR-345-5p and FZD5 transfection recovered colony formation ability (Fig. 6C, D). Certainly, cell apoptosis was notably stimulated by miR-345-5p transfection alone but repressed by miR-3455p + FZD5 transfection in ADM-treated THP-1 and HL-60 cells (Fig. 6E, F). In addition, cell cycle arrest was triggered in ADM-treated cells transfected with miR-345-5p but blocked in ADM-treated cells transfected with miR345-5p + FZD5 (Fig. 6G, H). Not surprisingly, cell migration and invasion were markedly restrained in ADM-treated cells with the transfection of miR-345-5p but promoted in ADM-treated cells with the transfection of miR-345-5p
+ FZD5 (Fig. 6I-L). Moreover, the expression of MMP2 and MMP9 was decreased by the transfection of miR-345$5 \mathrm{p}$ relative to miR-NC in ADM-treated cells but recovered by the transfection of miR-345-5p + FZD5 relative to miR$345-5 p+\operatorname{vector}$ (Fig. 6M, N). These functional analyses suggested that miR-345-5p mediated FZD5 to enhance the sensitivity of AML cells to ADM, thereby blocking the malignant properties of AML cells.

\section{Discussion}

Chemoresistance is one of the characteristics of AML that relapses after a brief remission following treatment, leading to resistance to further treatment [19]. Resistance to one drug can even lead to resistance to other drugs [20]. Therefore, exploring the mechanisms of development of drug resistance in AML and overcoming drug resistance will facilitate the treatment of AML. 
A $\begin{array}{ll}\text { FZD5 3' UTR WT } & \text { 5' aaaugagGCACAUACCGAGUCAGu 3' } \\ & \text { 3' cucgggaCCUG - AU-_-CCUCAGUCg 5, } \\ \text { miR-345-5p } & \text { 5' aaagagACGUACGCCACUGAGUu 3' } \\ \text { FZD5 3' UTR MUT } & \end{array}$

B

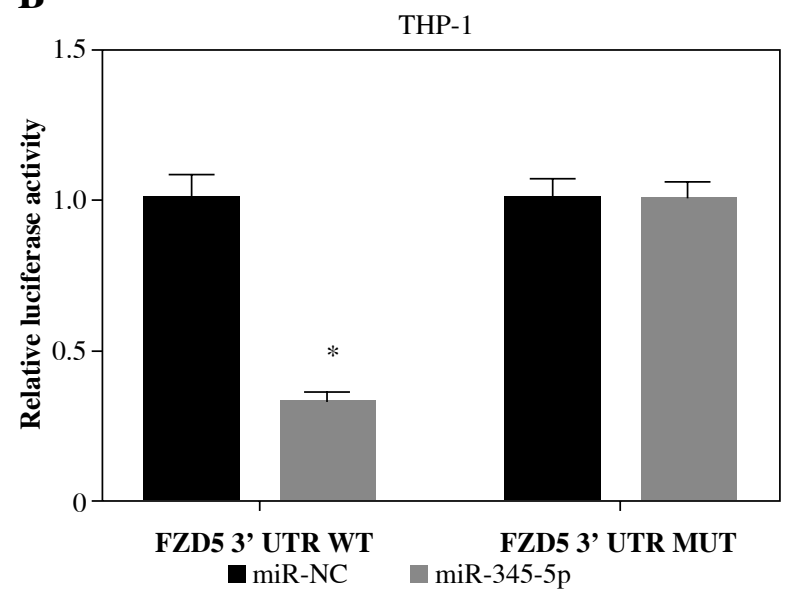

D

THP-1

FZD5

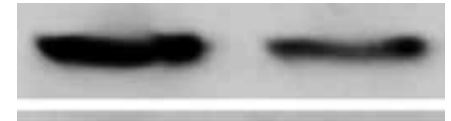

GAPDH

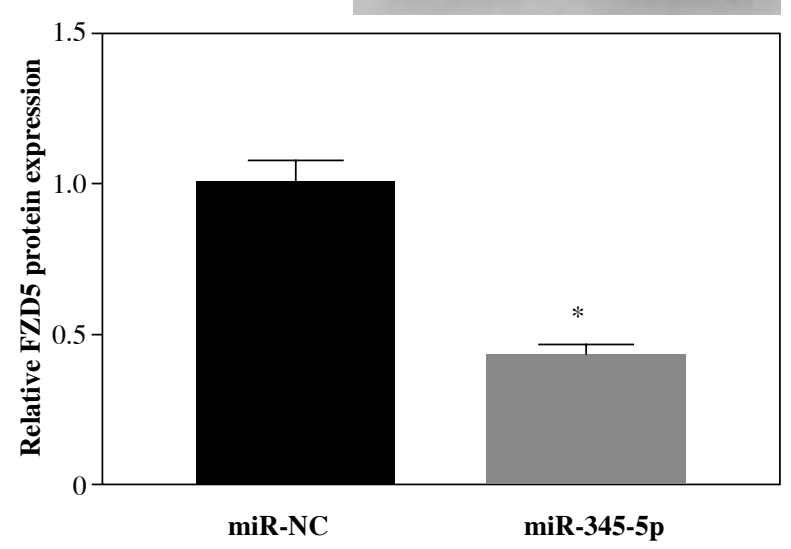

C

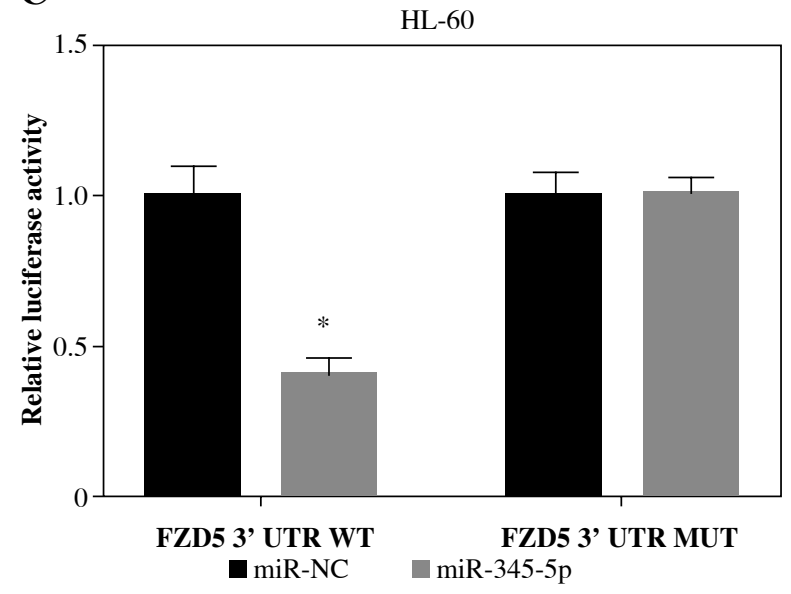

$\mathbf{E}$

HL-60

FZD5

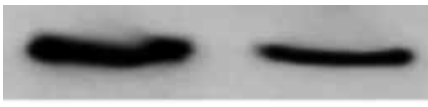

GAPDH

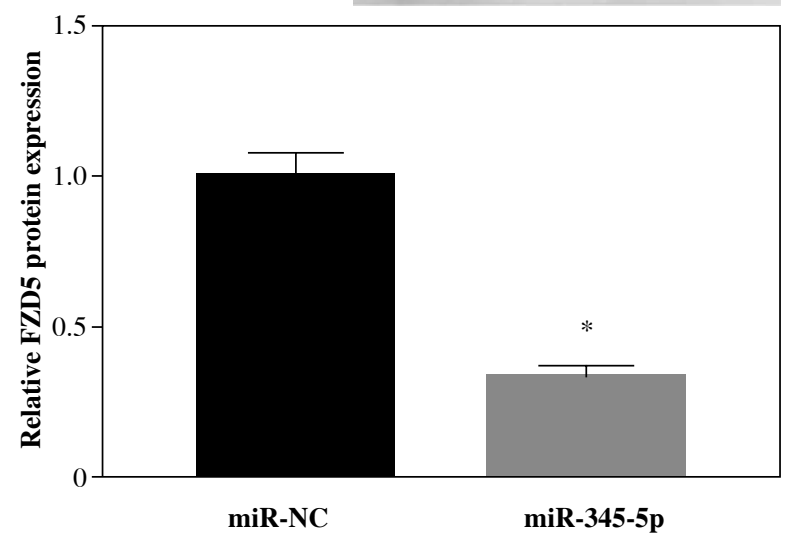

Fig. 5. FZD5 was a target of miR-345-5p and was regulated by circNPM1 and miR-345-5p. A) The interaction between FZD5 and miR-345-5p was predicted by the bioinformatics tool starBase. B, C) The interaction between FZD5 and miR345-5p was validated by dual-luciferase reporter assay. D, E) Expression of FZD5 in THP-1 and HL-60 cells with the transfection of miR-345-5p or miR-NC was measured by western blot. $* p<0.05$

Numerous circRNAs have been reported to be implicated in tumor initiation, chemoresistance to therapy, and hallmark, which was attributed to their abilities of gene regulation through diverse manners, including serving as microRNA sponges, and interacting with RNA-binding proteins [21, 22]. Recently, the mediation of circRNAs in drug resistance in AML attracts much attention. A previous study showed that circPAN3 was weakly expressed in chemo-sensitive AML patients compared to chemo-resistant AML patients, and circPAN3 knockdown suppressed the chemoresistance in AML cells to ADM by mediating the miR-153-5p/miR183-5p-XIAP pathway [23]. In this study, we concentrated on circNPM1 (circ_0075001), which was revealed to be upregulated in leukemia cell lines compared to healthy controls 
$\mathbf{F}$

F FD5

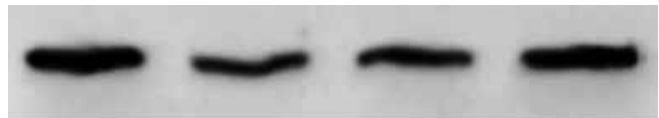

GAPDH

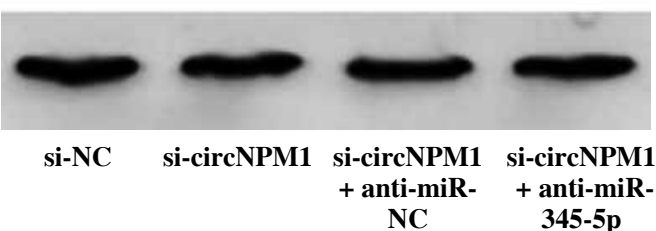

THP-1
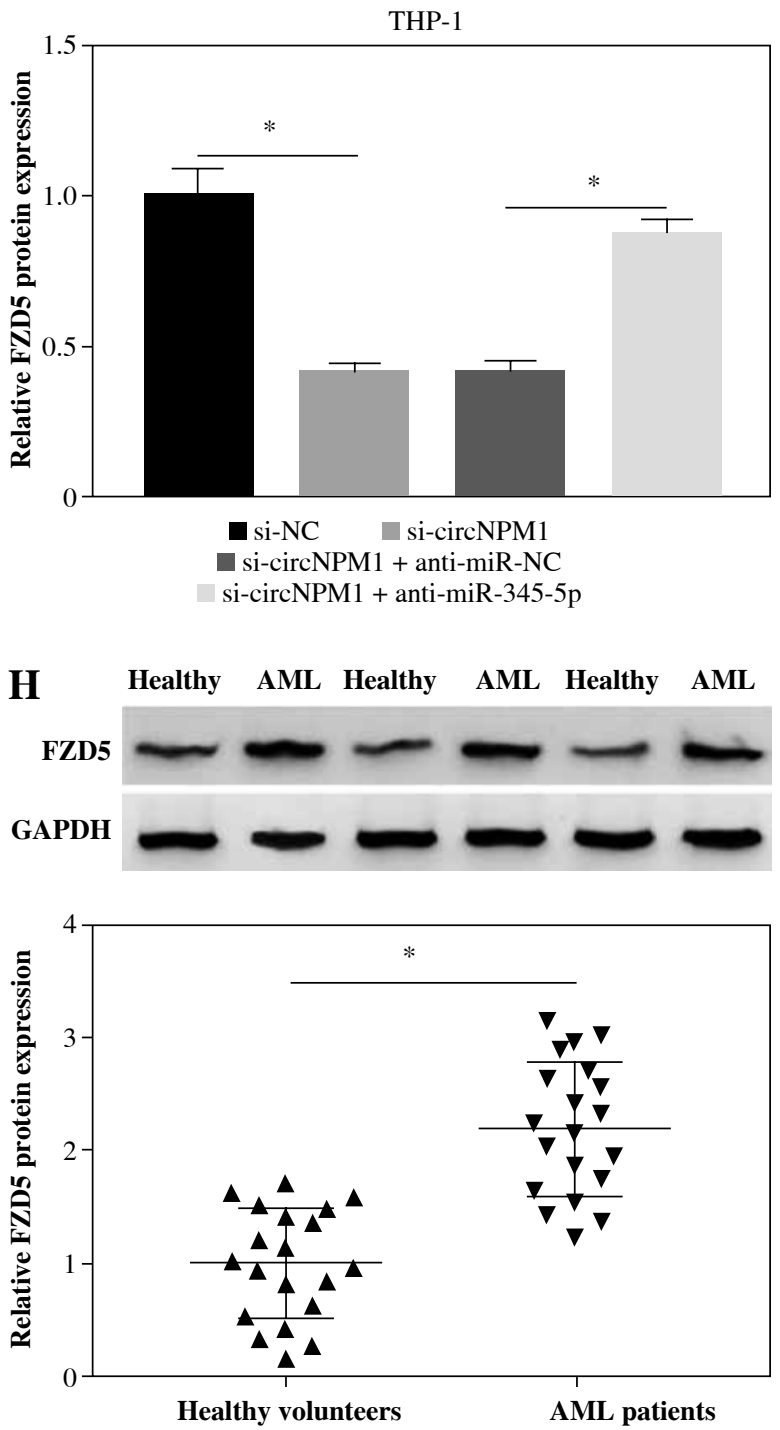

G

HL-60

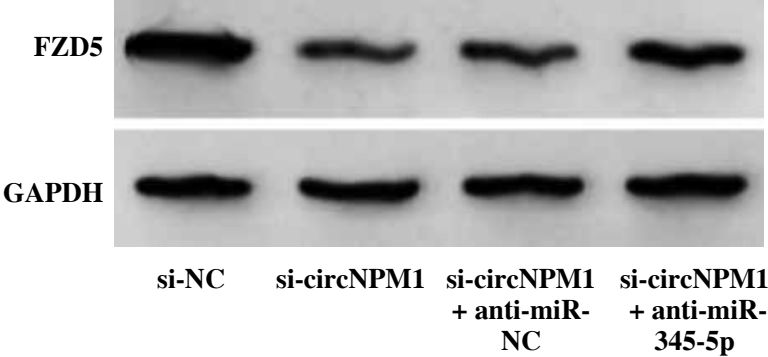

HL-60

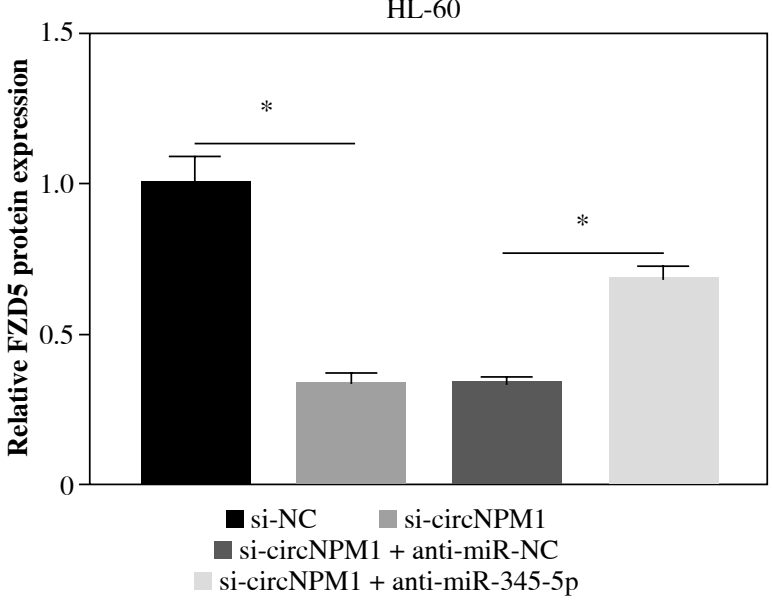

HS-5

THP-1

HL-60

I

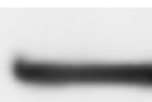

GAPDH
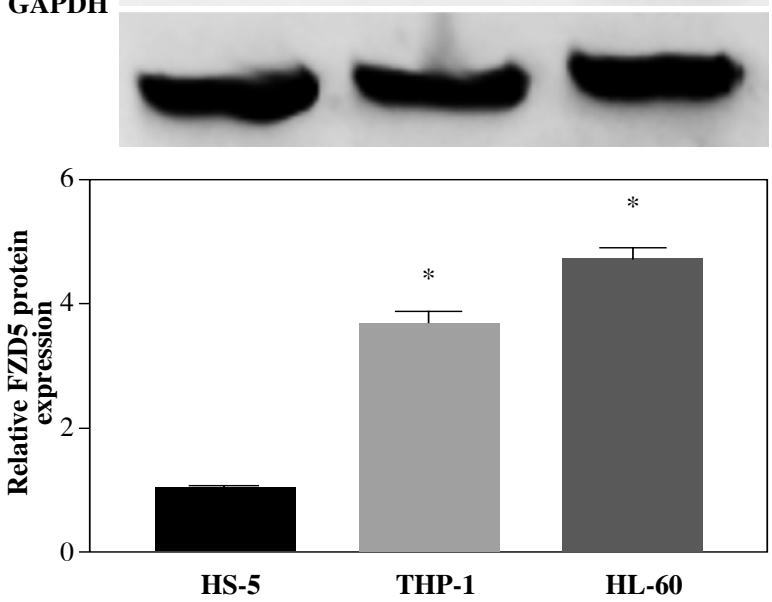

Fig. 5. Cont. F, G) Expression of FZD5 in THP-1 and HL-60 cells with the transfection of si-circNPM1, si-NC, si-circNPM1 + anti-miR-345-5p or si-circNPM1+anti-miR-NC was evaluated by western blot. H, I) Expression of FZD5 in serum samples and cell lines was detected by western blot. * $p<0.05$

[13]. The characteristics of circNPM1 were identified, and the data showed that circNPM1 was mainly located in the cytoplasm and resistant to RNase R. Functional experiments indicated that circNPM1 silence could strengthen the effects of $\mathrm{ADM}$, leading to further cell proliferation, migration and invasion inhibition, apoptosis promotion and cell cycle arrest. It suggested that circNPM1 might be an effective regulator of ADM chemoresistance in AML cells. 
A

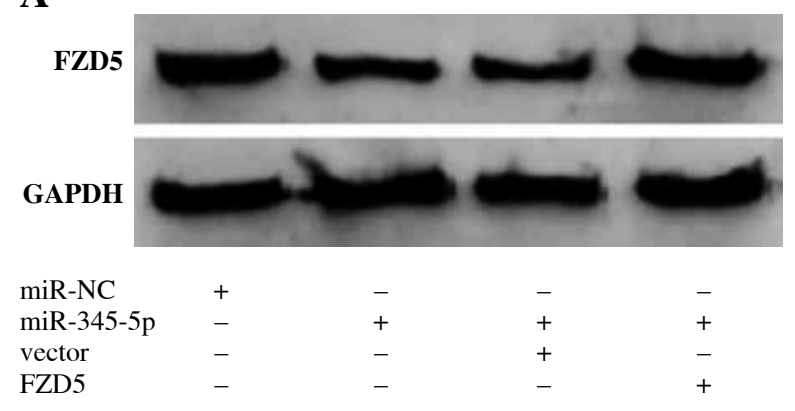

B

FZD5

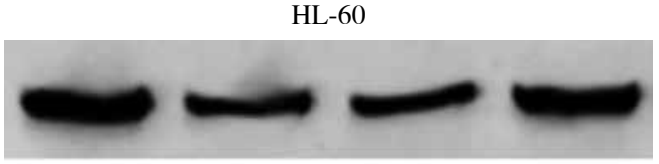

GAPDH
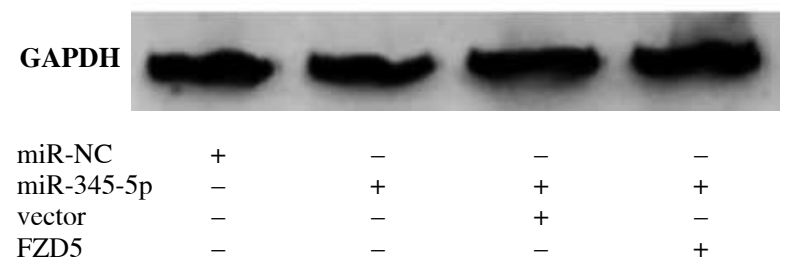

C

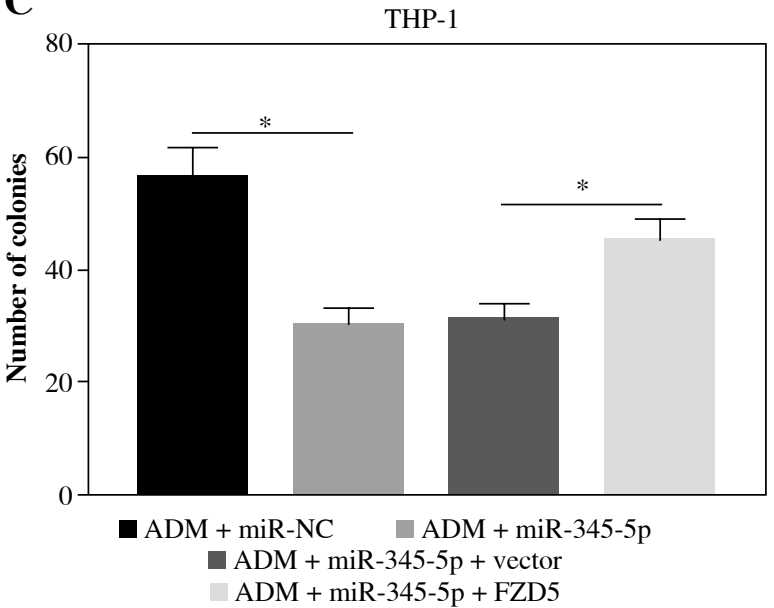

THP-1
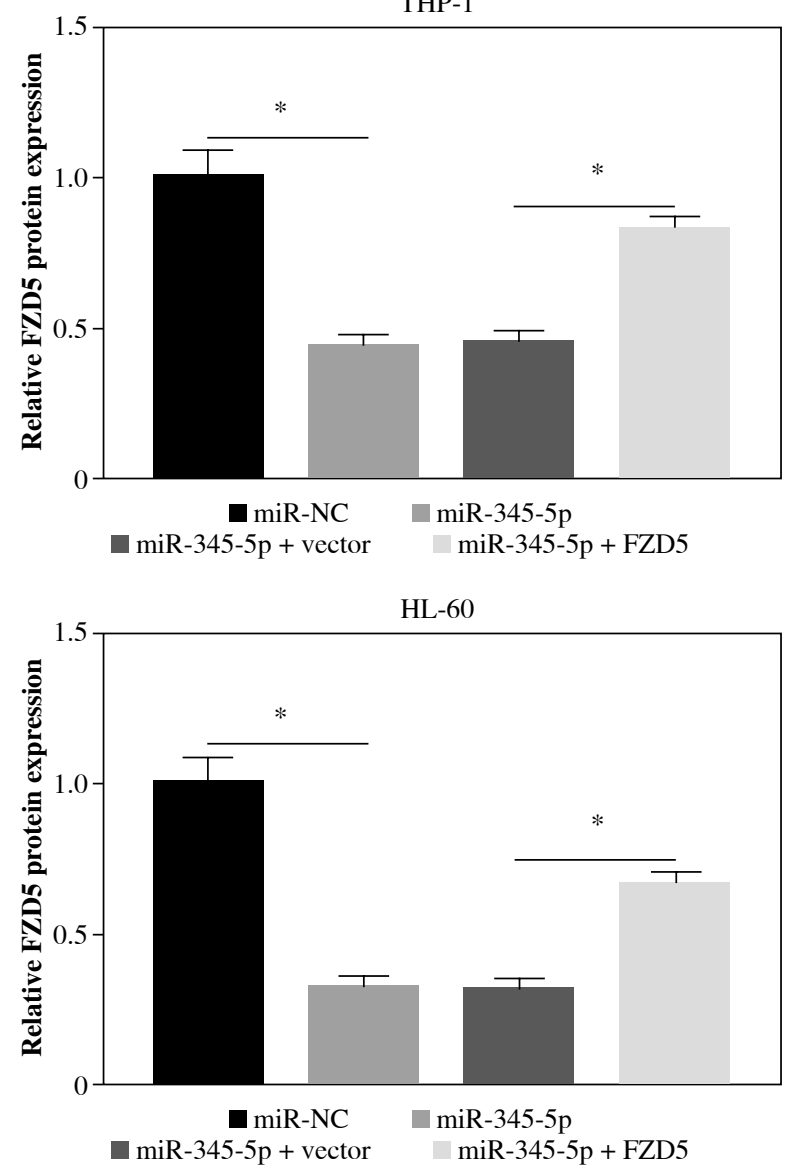

D

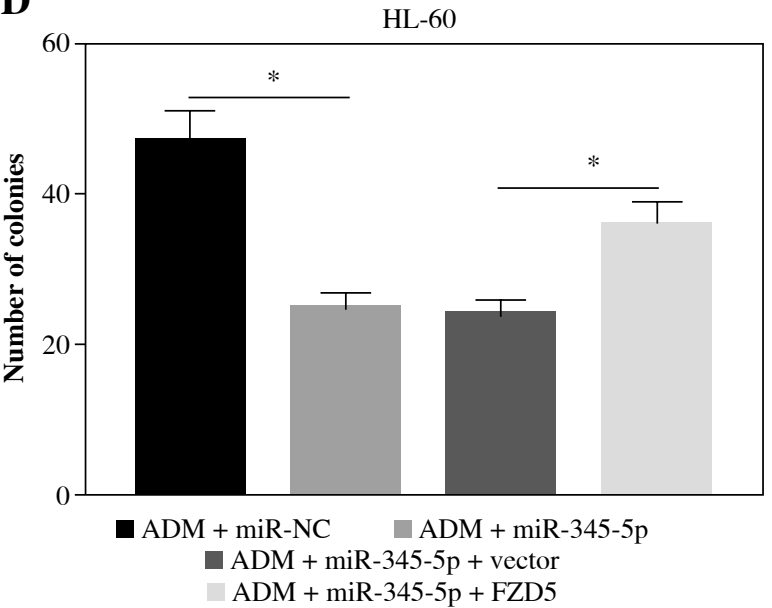

Fig. 6. FZD5 overexpression blocked the effects of miR-345-5p restoration in ADM-treated AML cells. THP-1 and HL-60 cells were transfected with miR-345-5p, miR-NC, miR-345-5p + FZD5 or miR-345-5p + vector. A, B) Expression of FZD5 in these transfected cells was detected by western blot to assess transfection efficiency. C, D) Cell proliferation was examined by colony formation assay. $* p<0.05$ 

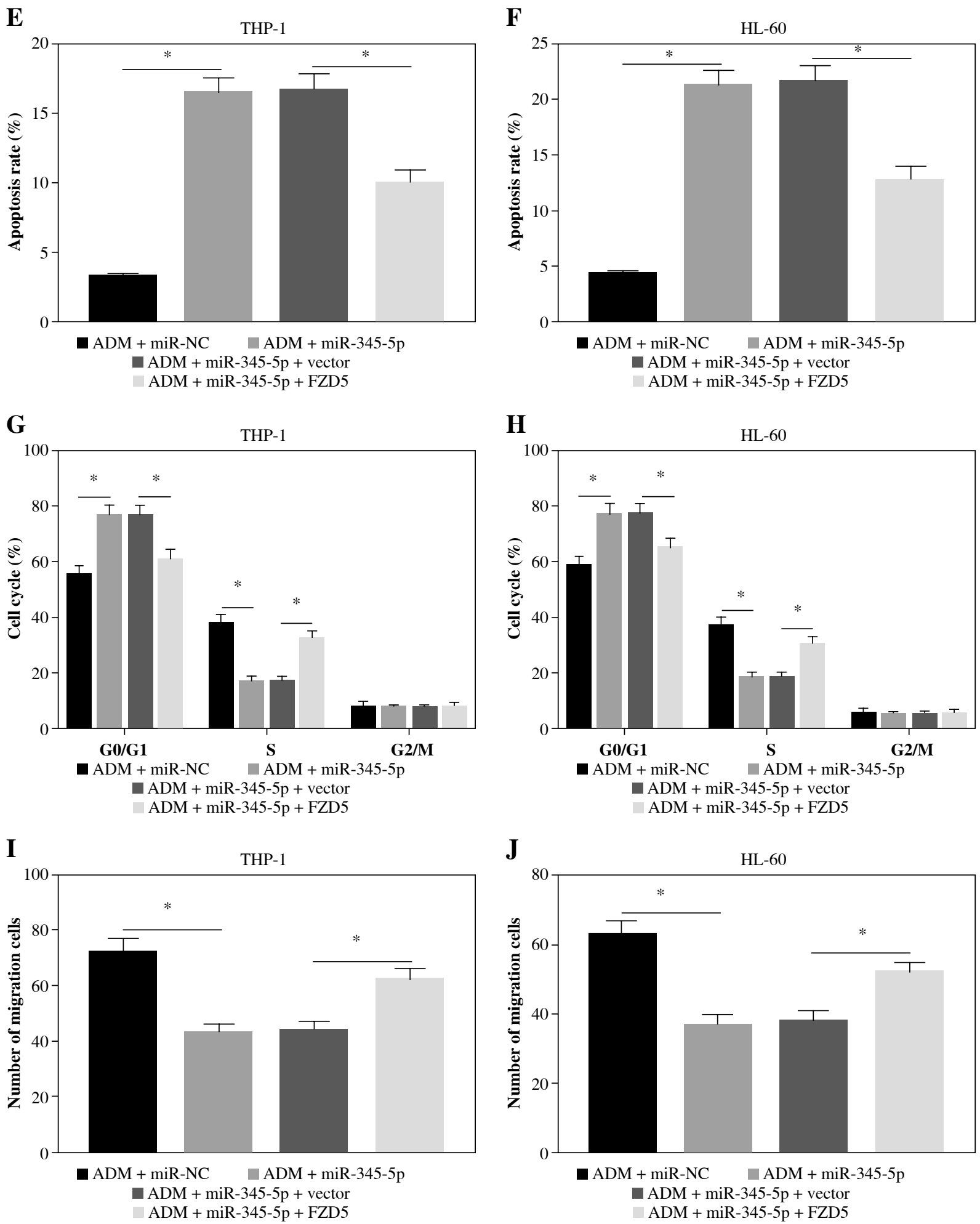

Fig. 6. Cont. E, F) Cell apoptosis was analyzed by flow cytometry assay. G, H) Cell cycle distribution was determined by flow cytometry assay. I, J) Cell migration and invasion were assessed by transwell assay. * $p<0.05$ 

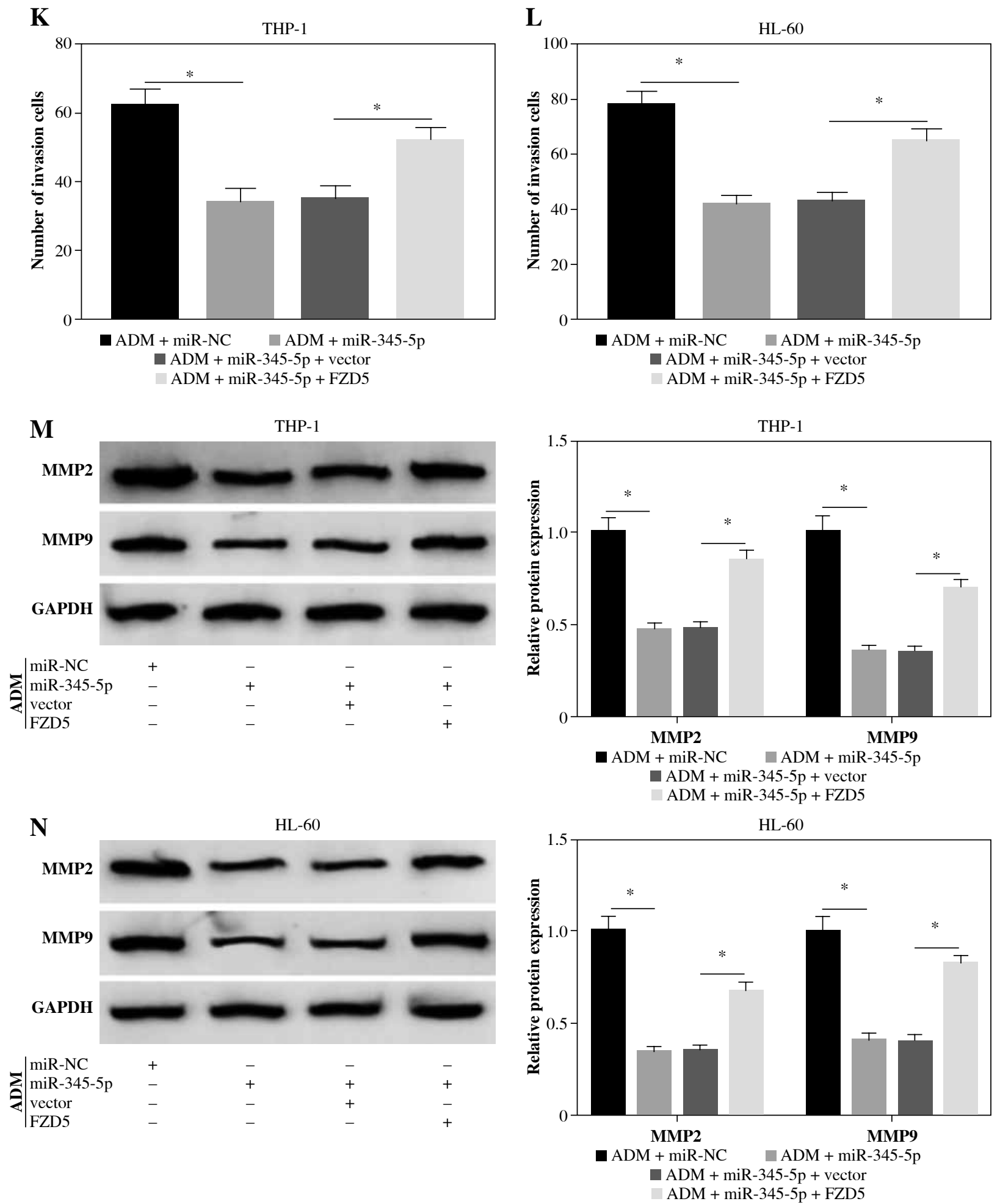

Fig. 6. Cont. K, L) Cell migration and invasion were assessed by transwell assay. M, N) Levels of MMP2 and MMP9 were quantified by western blot. ${ }^{*} p<0.05$ 
To explore the potential mechanism of circNPM1 action and ascertain whether circNPM1 acted as a miRNA sponge to play its biological functions, we screened and identified its putative target miRNAs by bioinformatics analysis, dual-luciferase reporter assay and RIP. Of these miRNAs, miR-345-5p was previously illustrated to be downregulated in AML, and miR-345-5p restoration frustrated cell proliferation and induced cell apoptosis [24]. Also, the expression of miR-345 was proved to be reduced in acute lymphocytic leukemia samples [25]. These consequences suggested the important role of miR-345-5p in hematological malignancy. In agreement with these ideas, we detected that the expression of miR-345-5p was decreased in AML serum samples and cell lines. MiR-345-5p absence could reverse the effects caused by circNPM1 silence, and miR-345-5p restoration promoted ADM sensitivity in AML cells to alleviate malignant cellular activities.

Further, we found that FZD5 was targeted by miR345-5p, and its expression was suppressed by miR-345$5 p$ restoration. In addition, the expression level of FZD5 was aberrantly elevated in AML serum samples and cell lines. FZD5 was regarded as an oncogene in various cancers, such as hepatocellular carcinoma, prostate cancer and ovarian cancer [26-28]. Also, FZD5 was targeted by miR-212-5p to reverse the effects of miR-212-5p mimic in AML cells, resulting in the promotion of proliferation and the block of apoptosis [29]. In our study, we first investigated the role of FZD5 in chemoresistance in AML cells and discovered that FZD5 overexpression could block the role of miR-345-5p restoration to increase ADM resistance in AML cells, thus promoting AML malignant development. Moreover, we found that expression of FZD5 was reduced in cells by the transfection of si-circNPM1, while the transfection of miR-345-5p inhibitor enhanced FZD5 expression, indicating that FZD5 was a downstream molecule of circNPM1 and miR-345-5p.

\section{Conclusions}

Collectively, this paper for the first time discussed the role of circNPM1 in drug resistance in AML cells and concluded that circNPM1 contributed to ADM chemoresistance in AML cells partly by mediating the miR-345-5p/FZD5 regulatory axis. Our study highlighted that circNPM1 might be a potential biomarker in drug resistance therapy for AML.

The authors declare no conflict of interest.

\section{References}

1. Dohner H, Weisdorf DJ, Bloomfield CD (2015): Acute myeloid leukemia. N Engl J Med 373: 1136-1152.

2. Gambacorta V, Gnani D, Vago L, et al. (2019): Epigenetic therapies for acute myeloid leukemia and their immune-related effects. Front Cell Dev Biol 7: 207.
3. Zhang J, Gu Y, Chen B (2019): Mechanisms of drug resistance in acute myeloid leukemia. Onco Targets Ther 12: 1937-1945.

4. Schiffer CA (2003): Hematopoietic growth factors and the future of therapeutic research on acute myeloid leukemia. N Engl J Med 349: 727-729.

5. Lokody I (2014): Drug resistance: overcoming resistance in acute myeloid leukaemia treatment. Nat Rev Cancer 14: 452-453.

6. Liu Y, Cheng Z, Pang Y, et al. (2019): Role of microRNAs, circRNAs and long noncoding RNAs in acute myeloid leukemia. J Hematol Oncol 12: 51.

7. Esteller M (2011): Non-coding RNAs in human disease. Nat Rev Genet 12: 861-874.

8. Zhang Z, Yang T, Xiao J (2018): Circular RNAs: Promising biomarkers for human diseases. EBioMedicine 34: 267-274.

9. Kristensen LS, Hansen TB, Veno MT, et al. (2018): Circular RNAs in cancer: opportunities and challenges in the field. Oncogene 37: 555-565.

10. Chen H, Liu T, Liu J, et al. (2018): Circ-ANAPC7 is upregulated in acute myeloid leukemia and appears to target the MiR-181 family. Cell Physiol Biochem 47: 1998-2007.

11. Yi YY, Yi J, Zhu X, et al. (2019): Circular RNA of vimentin expression as a valuable predictor for acute myeloid leukemia development and prognosis. J Cell Physiol 234: 3711-3719.

12. Wu DM, Wen X, Han XR, et al. (2018): Role of circular RNA DLEU2 in human acute myeloid leukemia. Mol Cell Biol 38: e00259-18.

13. Hirsch S, Blatte TJ, Grasedieck S, et al. (2017): Circular RNAs of the nucleophosmin (NPM1) gene in acute myeloid leukemia. Haematologica 102: 2039-2047.

14. Liao Q, Wang B, Li X, et al. (2017): miRNAs in acute myeloid leukemia. Oncotarget 8: 3666-3682.

15. Cammarata G, Augugliaro L, Salemi D, et al. (2010): Differential expression of specific microRNA and their targets in acute myeloid leukemia. Am J Hematol 85: 331-339.

16. Peterson YK, Nasarre P, Bonilla IV, et al. (2017): Frizzled-5: a high affinity receptor for secreted frizzled-related protein-2 activation of nuclear factor of activated T-cells c3 signaling to promote angiogenesis. Angiogenesis 20: 615-628.

17. Wright SC, Canizal MCA, Benkel T, et al. (2018): FZD5 is a Galphaq-coupled receptor that exhibits the functional hallmarks of prototypical GPCRs. Sci Signal 11: eaar5536.

18. Hua Y, Yang Y, Li Q, et al. (2018): Oligomerization of Frizzled and LRP5/6 protein initiates intracellular signaling for the canonical WNT/beta-catenin pathway. J Biol Chem 293: 19710-19724.

19. Jamal M, Song T, Chen B, et al. (2019): Recent progress on circular RNA research in acute myeloid leukemia. Front Oncol 9: 1108 .

20. Ullah MF (2008): Cancer multidrug resistance (MDR): a major impediment to effective chemotherapy. Asian Pac J Cancer Prev 9: 1-6.

21. Su M, Xiao Y, Ma J, et al. (2019): Circular RNAs in cancer: emerging functions in hallmarks, stemness, resistance and roles as potential biomarkers. Mol Cancer 18: 90.

22. Huang C, Shan G (2015): What happens at or after transcription: Insights into circRNA biogenesis and function. Transcription 6: 61-64.

23. Shang J, Chen WM, Wang ZH, et al. (2019): CircPAN3 mediates drug resistance in acute myeloid leukemia through the miR-153-5p/miR-183-5p-XIAP axis. Exp Hematol 70: $42-54$. 
24. Ying X, Zhang W, Fang M, et al. (2018): miR-345-5p regulates proliferation, cell cycle, and apoptosis of acute myeloid leukemia cells by targeting AKT2. J Cell Biochem.

25. Li X, Li D, Zhuang Y, et al. (2013): Overexpression of miR708 and its targets in the childhood common precursor B-cell ALL. Pediatr Blood Cancer 60: 2060-2067.

26. Thiele S, Rauner M, Goettsch C, et al. (2011): Expression profile of WNT molecules in prostate cancer and its regulation by aminobisphosphonates. J Cell Biochem 112: 1593-1600.

27. Zhu Q, Lu G, Luo Z, et al. (2018): CircRNA circ_0067934 promotes tumor growth and metastasis in hepatocellular carcinoma through regulation of miR-1324/FZD5/Wnt/beta-catenin axis. Biochem Biophys Res Commun 497: 626-632.

28. Bobbs A, Gellerman K, Hallas WM, et al. (2015): ARID3B directly regulates ovarian cancer promoting genes. PLoS One 10: e0131961.

29. Lin JF, Zeng H, Zhao JQ (2018): MiR-212-5p regulates the proliferation and apoptosis of AML cells through targeting FZD5. Eur Rev Med Pharmacol Sci 22: 8415-8422. 